\title{
Hall Effect on Radiative Casson Fluid Flow with Chemical Reaction on a Rotating Cone through Entropy Optimization
}

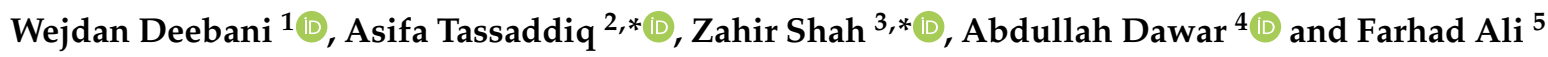 \\ 1 Department of Mathematics, College of Science and Arts, Rabigh King Abdul-Aziz University, \\ Rabigh 21911, Saudi Arabia; wdeebani@kau.edu.sa \\ 2 College of Computer and Information Sciences Majmaah University, Al Majmaah 11952, Saudi Arabia \\ 3 Center of Excellence in Theoretical and Computational Science (TaCS-CoE), SCL 802 Fixed Point Laboratory, \\ Science Laboratory Building, King Mongkut's University of Technology Thonburi (KMUTT), \\ Bangkok 10140, Thailand \\ 4 Department of Mathematics, Abdul Wali Khan University, Mardan 23200, Pakhtunkhwa, Pakistan; \\ abdullah.mathematician@gmail.com \\ 5 Department of Mathematics, City University of Science and Information Technology, \\ Peshawar 25000, KPK, Pakistan; farhadali@cusit.edu.pk \\ * Correspondence: a.tassaddiq@mu.edu.sa (A.T.); zahir.sha@kmutt.ac.th (Z.S.)
}

Received: 6 April 2020; Accepted: 20 April 2020; Published: 22 April 2020

\begin{abstract}
Magnetohydrodynamic (MHD) flow with Hall current has numerous applications in industrial areas such as Hall current accelerators, MHD power generators, planetary dynamics, Hall current sensors, etc. In this paper, the analysis of an unsteady MHD Casson fluid with chemical reaction over a rotating cone is presented. The impacts of Hall current, joule heating, thermal radiation, and viscous dissipation are analyzed. Entropy optimization is also considered in the present analysis. The system of coupled equations is tackled with homotopy analysis method (HAM). The convergence of HAM is also shown through figures. Deviations in the flow due to dimensionless parameters are shown graphically. Similarly, the variation in skin friction, Nusselt number, and Sherwood number are deliberated through Tables. A justification of the current consequences is presented.
\end{abstract}

Keywords: entropy; thermal radiation; casson fluid; hall effect; chemical reaction; rotating cone

\section{Introduction}

The fluid flows through a cone have inspired attention due to recent improvements of innovative technologies. Fluid flows have excellent applications in many engineering and industrial fields like aeronautical engineering, solar collectors, rotating heat exchangers, homeotherapy treatment, endoscopy scanning, electronic chips, etc. Keeping in mind the significance of rotating flows, Kumari et al. [1] first presented the convective flow through a vertical cone. Nadeem and Saleem [2] analyzed the unsteady convective magnetohydrodynamic (MHD) flow over a rotating cone. Hayat et al. [3] explored the irreversibility characterization of the convective fluid flow through a rotating cone. The MHD radiative flow with Soret and chemical reaction through a rotating cone was numerically analyzed by Sulochana et al. [4]. Raju and Sandeep [5] investigated the MHD bioconvective flow through a rotating cone. Zohraa et al. [6] probed the bioconvective nanofluid flow over a rotating cone. Raju and Sandeep [7] numerically analyzed the flow of a Casson fluid through a rotating cone. Nadeem and Saleem [8] analyzed the nanofluid through a rotating cone. The convective fluid flow with suction/injection over a rotating cone was presented by Ravindran et al. [9]. Chamkha and Al Mudhaf [10] investigated the mass and heat transmission in MHD fluid flow. 
The analysis of MHD flow with Hall currents has numerous applications in industrial areas such as Hall currents accelerators, MHD power generators, planetary dynamics, Hall current sensors, etc. Initially, Sato [11] investigated the Hall current influence on the ionized gas flow in parallel plates. Sherman and Sutton [12] presented the Hall current impact on MHD generator efficiency. Katagiri [13] examined the MHD boundary layer flow with Hall currents. Pop and Soundalgekar [14] determined the hydrodynamic flow with Hall current influence. Gupta [15] and Jana et al. [16] probed the Hall current effect on hydrodynamic flow over a plate. Pop and Watanabe [17] studied the MHD boundary layer flow with Hall current. Aziz [18] examined the thermal transmission in a fluid flow with Hall Effects. Seth and Singh [19] investigated the convective hydrodynamic flow with Hall current. Wahed [20] presented the MHD nanofluid flow with radiation and Hall current. Osalusi et al. [21] offered the unsteady MHD mixed convective flow with variable properties through a rotating cone.

Different fluids, such as viscoelastic fluid, Williamson fluid, Jeffrey fluid, micropolar fluid, power-law fluid, Casson fluid, etc., are named as non-Newtonian fluids. A model of the Casson fluid [22] is presented in 1959. The MHD Casson fluid flow under the influences of Dufour and Soret impacts was investigated by Hayat et al. [23]. Eldabe and Salwa [24] deliberated the MHD flow of Casson fluid in a rotating cylinder. The Casson fluid flow over an extending surface was determined by Malik et al. [25]. Aziz and Afify [26] presented the thermal transfer in MHD radiative Casson fluid. Shateyi et al. [27] observed the convective thermal and mass transmission in a Casson fluid flow with viscous dissipation effect. The Casson fluid flow with magnetic influence was determined by Shehzad et al. [28]. Reddy et al. [29] analyzed the thermal transmission in a Casson fluid flow over a thermal sheet. The above studies were based on the first law of thermodynamics.

It is well known that entropy, as a thermodynamic function, reflects a system's operating status. At the same time, it is necessary to minimize the entropy generation of a system to improve its working effectiveness. Entropy generation minimization techniques can be employed for the optimization of technical systems including heat exchangers, elements of nuclear and thermal power plants, ventilation and air conditioning systems, and so on. Thermodynamic second laws are utilized to examine the entropy optimization in term of the entropy age rate. Entropy augmentation is exploited to elucidate the performance of dissimilar contexts in modern and structure applications. Entropy is imitative from Greek word entropia, which implies that "a moving in the direction of" or "change". Entropy calculation is essential as it classifies the parameters for energy loss. Alternatively, thermodynamics second law was employed to minimalize the entropy optimization in engineering systems by Bejan [30,31]. Using different geometrical configurations, the authors of [32-36] have calculated the entropy generation. Chen et al. [37] and Liu and Lo [38] analyzed the entropy optimization in convective flow with viscous dissipation influence. Shah et al. $[39,40]$ recently investigated entropy optimization in a nanofluid flow in different geometries. Numerical and analytical approaches are used in their work for results. The others related investigations to MHD Casson fluid are mentioned in [39-46].

This work presents the analysis of MHD Casson fluid flow with chemical reaction over a rotating cone. Joule heating, radiation, viscous impact, and Hall current are deliberated in this work. Furthermore, the features of entropy generation with first-order chemical reaction are examined. The system of coupled equations is tackled with HAM. The convergence of HAM is also shown in the following figures. Deviation in the flow distributions due to dimensionless factors are published through figures. The validation of the present results is presented through tables. Table 1 led us to continue our analysis on Casson fluid flow with chemical reaction and entropy generation. 
Table 1. Studies on the fluid flows ( $\sqrt{ }=$ Effect is present, $\times=$ Effect is not present).

\begin{tabular}{ccccc}
\hline Reference \# & Fluid Type & Hall Current & Entropy Generation & Chemical Reaction \\
\hline Ref. [44] & Newtonian fluid & $\times$ & $\times$ & $\times$ \\
\hline Ref. [45] & Casson fluid & $\times$ & $\times$ & $\times$ \\
\hline Ref. [46] & Casson fluid & $\sqrt{ }$ & $\sqrt{ }$ & $\times$ \\
\hline Present study & Casson fluid & $\sqrt{ }$ & $\sqrt{ }$ & $\sqrt{ }$ \\
\hline
\end{tabular}

\section{Problem Modeling}

Unsteady and incompressible Casson fluid flow with chemical reaction by a rotating cone is considered here. The cone rotates with angular velocity $\Omega$ as shown in Figure 1 . The gravitational acceleration $g$ acts downward. Thermal radiation, Joule heating, and viscous dissipation are demonstrated in the temperature equation. Furthermore, the entropy generation is also considered.

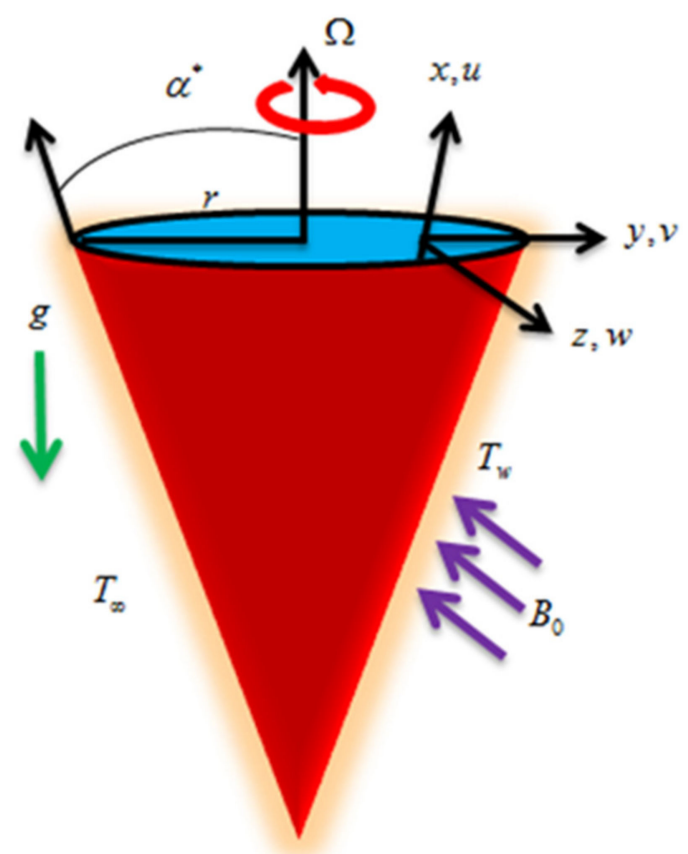

Figure 1. Fluid flow geometry.

The general Ohm's law with Hall current is defined as

$$
\vec{J}+\frac{\omega_{e} \tau_{e}}{B_{0}}(\vec{J} \times \vec{B})=\sigma(\vec{E}+\vec{V} \times \vec{B})
$$

Here, in this analysis, the electric field is ignored. Therefore, Equation (1) reduced as

$$
\begin{aligned}
& J_{x}=\frac{\sigma B_{0}}{\left(1+m^{2}\right)}(m u-v), \\
& J_{y}=\frac{\sigma B_{0}}{\left(1+m^{2}\right)}(m v+u),
\end{aligned}
$$

in which $m=\tau_{e} \omega_{e}$ represents the Hall factor. 
Furthermore, the basic model of the Casson fluid is demarcated as

$$
\tau_{i j}=\left\{\begin{array}{c}
2\left(\mu_{B}+\frac{S_{y}}{(2 \chi)^{1 / 2}}\right) e_{i j}, \quad x>\chi_{c} \\
2\left(\mu_{B}+\frac{S_{y}}{\left(2 \chi_{c}\right)^{1 / 2}}\right) e_{i j}, \quad x<\chi_{c}
\end{array}\right.
$$

where $\chi=e_{i j} e_{i j}$ is the product of deformation rate components, $\chi_{c}$ represents the critical value of this product, $\mu_{B}$ is the plastic dynamic viscosity, and $S_{y}$ is the fluid yield stress. When $\chi<\chi_{c}$, Equation (4) is summarized as

$$
\tau_{i j}=2 e_{i j}\left(1+\frac{1}{\psi}\right) \mu_{B}
$$

where $\psi=\frac{\left(2 \chi_{c}\right)^{1 / 2} \mu_{B}}{S_{y}}$ is the Casson factor.

In view of the mentioned assumptions, the leading equations of the Casson fluid and the general Ohm's law, including Hall current effect, are specified as

$$
\begin{gathered}
\frac{\partial(x u)}{\partial x}+\frac{\partial(x w)}{\partial z}=0 \\
\frac{\partial u}{\partial t}+u \frac{\partial u}{\partial x}-\frac{v^{2}}{x}+w \frac{\partial u}{\partial z}=v\left(1+\frac{1}{\psi}\right) \frac{\partial^{2} u}{\partial z^{2}}-\frac{\sigma B_{0}^{2}}{\rho\left(1+m^{2}\right)}(u+m v) \\
+g \beta_{t} \cos \alpha^{*}\left(T-T_{\infty}\right)+g \beta_{c} \cos \alpha^{*}\left(C-C_{\infty}\right), \\
\frac{\partial v}{\partial t}+u \frac{\partial v}{\partial x}+\frac{u v}{x}+w \frac{\partial v}{\partial z}=v\left(1+\frac{1}{\psi}\right) \frac{\partial^{2} v}{\partial z^{2}}+\frac{\sigma B_{0}^{2}}{\rho\left(1+m^{2}\right)}(m u-v), \\
\frac{\partial T}{\partial t}+u \frac{\partial T}{\partial x}+w \frac{\partial T}{\partial z}=\frac{k}{\rho c_{p}} \frac{\partial^{2} T}{\partial z^{2}}+\frac{\mu}{\rho c_{p}}\left(1+\frac{1}{\psi}\right)\left[\left(\frac{\partial u}{\partial z}\right)^{2}+\left(\frac{\partial v}{\partial z}\right)^{2}\right]+ \\
\frac{16}{3} \frac{\sigma^{*} T_{\infty}^{3}}{k^{*} \rho c_{p}}+\frac{\sigma B_{0}^{2}}{\rho c_{p}}\left(u^{2}+v^{2}\right), \\
\frac{\partial C}{\partial t}+u \frac{\partial C}{\partial x}+w \frac{\partial C}{\partial z}=D_{B} \frac{\partial^{2} C}{\partial z^{2}}-K_{r}\left(C-C_{\infty}\right),
\end{gathered}
$$

with

$$
\begin{aligned}
& \left.\begin{array}{c}
u(x, \zeta, t)=N_{0} \mu \frac{\partial u}{\partial z}, v(x, \zeta, t)=\frac{\Omega x \sin \alpha^{*}}{(1-q t)}+N_{0} \mu \frac{\partial v}{\partial z}, \\
w(x, \zeta, t)=0, T(x, \zeta, t)=T_{w}, C(x, \zeta, t)=C_{w}
\end{array}\right\} \text { at } \zeta=0, \\
& \left.u(x, \zeta, t)=0, v(x, \zeta, t)=0, T(x, \zeta, t)=T_{\infty}, C(x, \zeta, t)=C_{\infty}\right\} \text { at } \zeta=\infty .
\end{aligned}
$$

Here $x, y$ and $z ; u, v$ and $w$; and $\mu, \rho, \alpha^{*}, \beta_{t}, \beta_{c}, N_{0}, T, T_{w}, T_{\infty}, \sigma^{*}, c_{p}, C, C_{w}, C_{\infty}, D_{B}, \Omega$, and $K_{r}$ are the coordinate axes, respectively; velocity components, correspondingly; dynamic viscosity, density, semi-vertical angle, thermal coefficient, concentration coefficient, velocity slip factor, temperature, temperature at the surface, temperature away from the surface, Stefan-Boltzman constant, specific heat, concentration, concentration at the surface, concentration away from the surface, mass diffusivity, dimensionless angular velocity, and chemical reaction rate, respectively.

Considering

$$
\begin{gathered}
u=-\frac{1}{2} \frac{\Omega x \sin \alpha^{*}}{\left(1-q t^{*}\right)} f^{\prime}(\zeta), \quad v=\frac{\Omega x \sin \alpha^{*}}{\left(1-q t^{*}\right)} g(\zeta), w=\sqrt{\frac{v_{0} \Omega \sin \alpha^{*}}{\left(1-q t^{*}\right)}} f(\zeta), \\
\theta(\zeta)=\frac{T-T_{\infty}}{T_{w}-T_{\infty}}, \quad\left(T_{w}-T_{\infty}\right)=\frac{\left(T_{0}-T_{\infty}\right)}{\left(1-q t^{*}\right)^{2}} \frac{x}{L}, \quad \phi(\zeta)=\frac{C-C_{\infty}}{C_{w}-C_{\infty}}, \\
\left(C_{w}-C_{\infty}\right)=\frac{\left(C_{0}-C_{\infty}\right)}{\left(1-q t^{*}\right)^{2}} \frac{x}{L}, \quad t^{*}=\left(\Omega \sin \alpha^{*}\right) t, \quad \zeta=\sqrt{\frac{\Omega \sin \alpha^{*}}{\left(1-q t^{*}\right) v_{0}}} z .
\end{gathered}
$$


The transformed equations are

$$
\begin{gathered}
\left(1+\frac{1}{\psi}\right) f^{\prime \prime \prime}(\zeta)+\frac{1}{2} f^{\prime 2}(\zeta)-2 g^{2}(\zeta)-f(\zeta) f^{\prime \prime}(\zeta)-S\left(f^{\prime}(\zeta)+\frac{\zeta}{2} f^{\prime \prime}(\zeta)\right) \\
-2 \lambda(\theta(\zeta)+N \phi(\zeta))-\frac{M}{1+m^{2}}\left(f^{\prime}(\zeta)+m g(\zeta)\right)=0 \\
\left(1+\frac{1}{\psi}\right) g^{\prime \prime}(\zeta)+g(\zeta) f^{\prime}(\zeta)-f(\zeta) g^{\prime}(\zeta)- \\
S\left(g(\zeta)+\frac{\zeta}{2} g^{\prime}(\zeta)\right)+\frac{M}{1+m^{2}}\left(m f^{\prime}(\zeta)-g(\zeta)\right)=0 \\
(1+R d) \theta^{\prime \prime}(\zeta)+\frac{1}{2} \operatorname{Pr} f^{\prime}(\zeta) \theta(\zeta)-\operatorname{Pr} f(\zeta) \theta^{\prime}(\zeta)-\operatorname{Pr} S\left(2 \theta(\zeta)+\frac{\zeta}{2} \theta^{\prime}(\zeta)\right) \\
+B r\left(1+\frac{1}{\psi}\right)\left(\frac{1}{4} f^{\prime \prime}{ }^{2}(\zeta)+g^{\prime 2}(\zeta)\right)+M B r\left(\frac{1}{4} f^{\prime 2}(\zeta)+g^{2}(\zeta)\right)=0, \\
\phi^{\prime \prime}(\zeta)+\frac{1}{2} S c f^{\prime}(\zeta) \phi(\zeta)-S\left(2 \phi(\zeta)+\frac{\zeta}{2} \phi^{\prime}(\zeta)\right)-S c f(\zeta) \phi^{\prime}(\zeta)-\gamma S c \phi(\zeta)=0, \\
f(\zeta)=0, f^{\prime}(\zeta)=\beta f^{\prime \prime}(\zeta), g(\zeta)=1+\beta g^{\prime}(\zeta), \theta(\zeta)=1, \phi(\zeta)=1 \text { at } \zeta=0, \\
f^{\prime}(\zeta) \rightarrow 0, g(\zeta) \rightarrow 0, \theta(\zeta) \rightarrow 0, \phi(\zeta) \rightarrow 0 \text { as } \zeta \rightarrow \infty .
\end{gathered}
$$

where Re, $G r, \operatorname{Pr}, E c, B r$, and $S c$ denote the Reynolds, Grashof, Prandtl, Eckert, Brinkman, and Schmidt numbers, respectively. $M, \lambda, N, \beta, R d$, and $\gamma$ are magnetic, mixed convection, buoyancy ratio, velocity slip, radiation, and chemical reaction parameters, respectively, which are defined as

$$
\begin{gathered}
\operatorname{Re}=\frac{L^{2} \Omega \sin \alpha^{*}}{v}, G r=\frac{g \beta_{t} \cos \alpha^{*}\left(T_{0}-T_{\infty}\right) L^{3}}{v^{2}}, M=\frac{\sigma B_{0}^{2}}{\rho \Omega \sin \alpha^{*}}, \\
S c=\frac{v}{D}, N=\frac{\beta_{c}\left(C_{0}-C_{\infty}\right)}{\beta_{t}\left(T_{0}-T_{\infty}\right)}, \lambda=\frac{G r}{\operatorname{Re}^{2}}, B r=\operatorname{Pr} E c, \gamma=\frac{k_{r}}{\Omega \sin \alpha^{*}}, \\
\beta=N_{0} \sqrt{\frac{\Omega \sin \alpha^{*}}{v}}, \operatorname{Pr}=\frac{v_{0}}{\alpha}, E c=\frac{\Omega^{2} L x \sin ^{2} \alpha^{*}}{c_{p}\left(T_{0}-T_{\infty}\right)}, R d=\frac{16}{3} \frac{\sigma^{*} T_{\infty}^{3}}{k k^{*}} .
\end{gathered}
$$

Velocity, Temperature, and Mass Gradients

The velocity $\left(C_{f x}, C_{f y}\right)$, temperature $\left(N u_{x}\right)$, and mass $\left(S h_{x}\right)$ gradients are

$$
C_{f x}=\frac{\left.2 \tau_{x z}\right|_{z=0}}{\rho\left(\frac{\Omega x \sin \alpha^{*}}{1-s t^{*}}\right)^{2}}, \quad C_{f y}=\frac{\left.2 \tau_{y z}\right|_{z=0}}{\rho\left(\frac{\Omega x \sin \alpha^{*}}{1-s t^{*}}\right)^{2}}, N u_{x}=\frac{\left.x q_{w}\right|_{z=0}}{k\left(T_{w}-T_{\infty}\right)}, S h_{x}=\frac{\left.x h_{w}\right|_{z=0}}{k\left(C_{w}-C_{\infty}\right)},
$$

where $\tau_{x z}$ and $\tau_{y z}$ are shear stresses, $q_{w}$ and $h_{w}$ are heat and mass fluxes, which are defined as

$$
\begin{gathered}
\tau_{x z}=-\left(\mu_{B}+\frac{S_{y}}{\left(2 \chi_{c}\right)^{1 / 2}}\right)\left(\frac{\partial u}{\partial z}\right), \tau_{y z}=-\left(\mu_{B}+\frac{S_{y}}{\left(2 \chi_{c}\right)^{1 / 2}}\right)\left(\frac{\partial v}{\partial z}\right), \\
q_{w}=-\left(\frac{\partial T}{\partial z}\right)-\frac{16}{3} \frac{\sigma^{*} T_{\infty}^{3}}{k^{*}}\left(\frac{\partial T}{\partial z}\right), h_{w}=-D\left(\frac{\partial C}{\partial z}\right) .
\end{gathered}
$$

The dimensionless forms are

$$
\begin{gathered}
C_{f x}\left(\operatorname{Re}_{x}\right)^{1 / 2}=-\left(1+\frac{1}{\psi}\right) f^{\prime \prime}(0), \quad \frac{1}{2} C_{f y}\left(\operatorname{Re}_{x}\right)^{1 / 2}=-\left(1+\frac{1}{\psi}\right) g^{\prime}(0), \\
\frac{N u_{x}}{\left(\operatorname{Re}_{x}\right)^{1 / 2}}=-(1+R d) \theta^{\prime}(0), \frac{S h_{x}}{\left(\operatorname{Re}_{x}\right)^{1 / 2}}=-\phi^{\prime}(0) .
\end{gathered}
$$




\section{Entropy Optimization}

Mathematical expression of entropy optimization is

$$
\begin{aligned}
S_{G}= & \frac{k}{T_{\infty}^{2}}\left(1+\frac{16}{3} \frac{\sigma^{*} T_{\infty}^{3}}{k^{*} k}\right)\left(\frac{\partial T}{\partial z}\right)^{2}+\frac{\mu_{B}}{T_{\infty}}\left(1+\frac{1}{\psi}\right)\left[\left(\frac{\partial u}{\partial z}\right)^{2}+\left(\frac{\partial v}{\partial z}\right)^{2}\right] \\
& +\frac{R_{D}}{T_{\infty}}\left(\frac{\partial C}{\partial z} \frac{\partial T}{\partial z}\right)+\frac{R_{D}}{C_{\infty}}\left(\frac{\partial C}{\partial z}\right)^{2}+\frac{\mu_{B}}{T_{\infty}} \frac{\sigma B_{0}^{2}}{\rho\left(1+m^{2}\right)}\left(u^{2}+v^{2}\right),
\end{aligned}
$$

The dimensionless form is

$$
\begin{gathered}
N_{G}=T_{d}(1+R d)\left(\theta^{\prime}(\zeta)\right)^{2}+\frac{B r}{A}\left(1+\frac{1}{\psi}\right)\left(\frac{1}{4} f^{\prime \prime 2}(\zeta)+g^{\prime 2}(\zeta)\right)+ \\
L \theta^{\prime}(\zeta) \phi^{\prime}(\zeta)+L \frac{C_{d}}{T_{d}} \phi^{\prime 2}(\zeta)+\frac{M B r}{A\left(1+m^{2}\right)}\left(\frac{1}{4} f^{\prime 2}(\zeta)+g^{2}(\zeta)\right)
\end{gathered}
$$

Bejan Number

The Bejan number is defined as

$$
\begin{gathered}
B e=\frac{\text { Irreversibilities due to heat and mass transfer }}{\text { Total entropy rate }}, \\
B e=\frac{T_{d}(1+R d)\left(\theta^{\prime}(\zeta)\right)^{2}+L \theta^{\prime}(\zeta) \phi^{\prime}(\zeta)+L \frac{C_{d}}{T_{d}} \phi^{\prime 2}(\zeta)}{\left[\begin{array}{c}
T_{d}(1+R d)\left(\theta^{\prime}(\zeta)\right)^{2}+\frac{B r}{A}\left(1+\frac{1}{\psi}\right)\left(\frac{1}{4} f^{\prime \prime}{ }^{2}(\zeta)+g^{\prime 2}(\zeta)\right)+ \\
L \theta^{\prime}(\zeta) \phi^{\prime}(\zeta)+L \frac{C_{d}}{T_{d}} \phi^{\prime 2}(\zeta)+\frac{M B r}{A\left(1+m^{2}\right)}\left(\frac{1}{4} f^{\prime 2}(\zeta)+g^{2}(\zeta)\right)
\end{array}\right]} .
\end{gathered}
$$

where $N_{G}$ signifies the entropy rate; $L$ indicates the diffusion parameter; $T_{d}$ and $C_{d}$ represent temperature difference and concentration difference, respectively; and $A$ is the dimensionless parameter which are defined as

$$
N_{G}=\frac{v_{0} T_{\infty} S_{G} L^{2}}{k\left(T_{0}-T_{\infty}\right) \Omega x^{2} \sin \alpha^{*}}, L=\frac{\left(C_{0}-C_{\infty}\right) R_{D}}{k}, T_{d}=\frac{T_{0}-T_{\infty}}{T_{\infty}}, C_{d}=\frac{C_{0}-C_{\infty}}{C_{\infty}}, A=\frac{x}{L} .
$$

\section{HAM Solution}

The analytical solution of the modeled coupled equations given in Equations (13-16) is conceded by HAM. It is supposed that $f=U, g=V, \theta=T$ and $\phi=C$. The initial suppositions and linear operatives for velocities, temperature, and concentration functions, respectively, are given as

$$
\begin{gathered}
U_{0}(\zeta)=0, V_{0}(\zeta)=\frac{1}{1+\beta} e^{-\zeta}, T_{0}(\zeta)=e^{-\zeta}, C_{0}(\zeta)=e^{-\zeta} . \\
L_{U}(U)=U^{\prime \prime \prime}-U^{\prime}, L_{V}(V)=V^{\prime \prime}-V, L_{T}(T)=T^{\prime \prime}-T, L_{C}(C)=C^{\prime \prime}-C .
\end{gathered}
$$

With

$$
\begin{gathered}
L_{U}\left(\bar{Y}_{1}+\bar{Y}_{2} e^{-\zeta}+\bar{Y}_{3} e^{\zeta}\right)=0, L_{V}\left(\bar{Y}_{4} e^{-\zeta}+\bar{Y}_{5} e^{\zeta}\right)=0, \\
L_{T}\left(\bar{Y}_{6} e^{-\zeta}+\bar{Y}_{7} e^{\zeta}\right)=0, L_{C}\left(\bar{Y}_{8} e^{-\zeta}+\bar{Y}_{9} e^{\zeta}\right)=0 .
\end{gathered}
$$

where $\bar{Y}_{1}, \bar{Y}_{2}, \bar{Y}_{3}, \ldots, \bar{Y}_{9}$ are constants.

$0^{\text {th }}-$ order problems

Let $\in[0,1]$ and $\hbar_{U}, \hbar_{V}, \hbar_{T}, \hbar_{C}$ be the embedding and non-zero auxiliary factors, respectively, then

$$
\begin{gathered}
(1-) L_{U}\left[U\left(\zeta_{;}\right)-U_{0}()\right]=\hbar_{U} \boldsymbol{\aleph}_{U}\left[U\left(\zeta_{;}\right), V\left(\zeta_{;}\right), T\left(\zeta_{;}\right), C\left(\zeta_{;}\right)\right], \\
(1-) L_{V}\left[V\left(\zeta_{;}\right)-V_{0}(\zeta)\right]=\hbar_{V} \boldsymbol{\aleph}_{V}\left[V\left(\zeta_{;}\right), U\left(\zeta_{;}\right)\right]
\end{gathered}
$$




$$
\begin{aligned}
& (1-) L_{T}\left[T\left(\zeta_{i}\right)-T_{0}(\zeta)\right]=\hbar_{T} \boldsymbol{\aleph}_{T}\left[T\left(\zeta_{i}\right), U\left(\zeta_{i}\right), V\left(\zeta_{i}\right)\right], \\
& (1-) L_{C}\left[C\left(\zeta_{;}\right)-C_{0}(\zeta)\right]=\hbar_{C} \boldsymbol{\kappa}_{C}\left[C\left(\zeta_{;}\right), U\left(\zeta_{i}\right)\right] \text {, } \\
& U(0 ;)=0, U^{\prime}(0 ;)=\beta U^{\prime \prime}(0 ;), \\
& V(0 ;)=1+\beta V^{\prime}(0 ;), T(0 ;)=C(0 ;)=1 \\
& U^{\prime}(\infty ;)=V(\infty ;)=0, T(\infty ;)=C(\infty ;)=0 \text {, } \\
& \boldsymbol{\aleph}_{U}\left[U\left(\zeta_{;}\right), V\left(\zeta_{;}\right), T\left(\zeta_{i}\right), C\left(\zeta_{;}\right)\right]=\left(1+\frac{1}{\psi}\right) \frac{\partial^{3} U\left(\zeta_{;}\right)}{\partial \zeta^{3}}+\frac{1}{2}\left(\frac{\partial U\left(\zeta_{;}\right)}{\partial \zeta}\right)^{2} \\
& -2\left(V\left(\zeta_{i}\right)\right)^{2}-U\left(\zeta_{;}\right) \frac{\partial^{2} U\left(\zeta_{;}\right)}{\partial \zeta^{2}}-S\left(\frac{\partial U\left(\zeta_{;}\right)}{\partial \zeta}+\frac{1}{2} \zeta \frac{\partial^{2} U\left(\zeta_{;}\right)}{\partial \zeta^{2}}\right) \\
& -2 \lambda\left(T\left(\zeta_{i}\right)+N C\left(\zeta_{i}\right)\right)-\frac{M}{1+m^{2}}\left(\frac{\partial U\left(\zeta_{;}\right)}{\partial \zeta}+m V\left(\zeta_{;}\right)\right), \\
& -S\left(V\left(\zeta_{i}\right)+\frac{1}{2} \zeta \frac{\partial V\left(\zeta_{i}\right)}{\partial \zeta}\right)+\frac{M}{1+m^{2}}\left(m \frac{\partial U\left(\zeta_{;}\right)}{\partial \zeta}-V\left(\zeta_{;}\right)\right), \\
& \boldsymbol{\aleph}_{T}\left[T\left(\zeta_{i}\right), U\left(\zeta_{i}\right), V\left(\zeta_{i}\right)\right]=(1+R d) \frac{\partial^{2} T\left(\zeta_{;}\right)}{\partial \zeta^{2}}+\frac{1}{2} \operatorname{Pr} T\left(\zeta_{i}\right) \frac{\partial U\left(\zeta_{;}\right)}{\partial \zeta} \\
& -\operatorname{Pr} U\left(\zeta_{i}\right) \frac{\partial T\left(\zeta_{;}\right)}{\partial \zeta}-\operatorname{SPr}\left(2 T(\zeta ;)+\frac{\zeta}{2} \frac{\partial T(\zeta ;)}{\partial \zeta}\right)+ \\
& B r\left(1+\frac{1}{\psi}\right)\left(\frac{1}{4}\left(\frac{\partial^{2} U\left(\zeta_{;}\right)}{\partial \zeta^{2}}\right)^{2}+\left(\frac{\partial V\left(\zeta_{;}\right)}{\partial \zeta}\right)^{2}\right)+\operatorname{MBr}\left(\frac{1}{4}\left(\frac{\partial U\left(\zeta_{;}\right)}{\partial \zeta}\right)^{2}+\left(V\left(\zeta_{;}\right)\right)^{2}\right), \\
& \boldsymbol{\aleph}_{C}\left[C\left(\zeta_{;}\right) ; U\left(\zeta_{;}\right)\right]=\frac{\partial^{2} C\left(\zeta_{;}\right)}{\partial \zeta^{2}}+\frac{1}{2} S c C\left(\zeta_{;}\right) \frac{\partial U\left(\zeta_{;}\right)}{\partial \zeta} \\
& -S c U\left(\zeta_{i}\right) \frac{\partial C\left(\zeta_{i}\right)}{\partial \zeta}-S\left(2 C\left(\zeta_{i}\right)+\frac{1}{2} \zeta \frac{\partial C\left(\zeta_{i}\right)}{\partial \zeta}\right)-\gamma S c C\left(\zeta_{i}\right) .
\end{aligned}
$$

$t^{\text {th }}-$ order problems are

$$
\begin{aligned}
& L_{U}\left[U_{t}(\zeta)-\chi_{t} U_{t-1}(\zeta)\right]=h_{t} \mathfrak{R}_{t}^{U}(\zeta), \\
& L_{V}\left[V_{t}(\zeta)-\chi_{t} V_{t-1}(\zeta)\right]=h_{V} \mathfrak{R}_{t}^{V}(\zeta), \\
& L_{T}\left[T_{t}(\zeta)-\chi_{t} T_{t-1}(\zeta)\right]=h_{T} \mathfrak{R}_{t}^{T}(\zeta), \\
& L_{C}\left[C_{t}(\zeta)-\chi_{t} C_{t-1}(\zeta)\right]=h_{C} \mathfrak{R}_{t}^{C}(\zeta), \\
& U_{t}(0)=U^{\prime}{ }_{t}(0)=U^{\prime}{ }_{t}(\infty)=0, \quad V_{t}(0)=V_{t}(\infty)=0, \quad T_{t}(0)=T_{t}(\infty)=0, \quad C_{t}(0)=C_{t}(\infty)=0 . \\
& \mathfrak{R}_{t}^{U}(\zeta)=\left(1+\frac{1}{\psi}\right) U^{\prime \prime \prime}{ }_{t-1}+\frac{1}{2} U^{\prime 2}{ }_{t-1}-2 V_{t-1}^{2}-\sum_{j=0}^{t-1}\left(U_{t-1-j} U^{\prime \prime}{ }_{j}\right)- \\
& S\left(U^{\prime}{ }_{t-1}+\frac{1}{2} \zeta U^{\prime \prime}{ }_{t-1}\right)-2 \lambda\left(T_{t-1}+N C_{t-1}\right)-\frac{M}{1+m^{2}}\left(U^{\prime}{ }_{t-1}+m V_{t-1}\right), \\
& \mathfrak{R}_{t}^{V}(\zeta)=\left(1+\frac{1}{\psi}\right) V^{\prime \prime}{ }_{t-1}+\sum_{j=0}^{t-1}\left(V_{t-1-j} U^{\prime}{ }_{j}\right)-\sum_{j=0}^{t-1}\left(U_{t-1-j} V^{\prime}{ }_{j}\right) \\
& -S\left(V_{t-1}+\frac{1}{2} \zeta V_{t-1}^{\prime}\right)+\frac{M}{1+m^{2}}\left(m U^{\prime}{ }_{t-1}-V_{t-1}\right), \\
& \mathfrak{R}_{t}^{T}(\zeta)=(1+R d) T^{\prime \prime}{ }_{t-1}+\frac{1}{2} \operatorname{Pr} \sum_{j=0}^{t-1}\left(U^{\prime}{ }_{t-1-j} T_{j}\right)-\operatorname{Pr} \sum_{j=0}^{t-1}\left(U_{t-1-j} T^{\prime}{ }_{j}\right)- \\
& \operatorname{Pr} S\left(2 T_{t-1}+\frac{\zeta}{2} T_{t-1}^{\prime}\right)+\operatorname{Br}\left(1+\frac{1}{\psi}\right)\left(\frac{1}{4} U_{t-1}^{\prime \prime 2}+V_{j}^{\prime 2}\right)+\operatorname{MBr}\left(\frac{1}{4} U_{t-1}^{\prime 2}+V_{t-1}^{2}\right), \\
& \mathfrak{R}_{t}^{C}(\zeta)=C^{\prime \prime}{ }_{t-1}+\frac{1}{2} S c \sum_{j=0}^{t-1}\left(U^{\prime}{ }_{t-1-j} C_{j}\right)-S\left(2 C_{t-1}+\frac{\zeta}{2} C^{\prime}{ }_{t-1}\right)-S c \sum_{j=0}^{t-1}\left(U_{t-1-j} C^{\prime}{ }_{j}\right)-\gamma S c C_{t-1} .
\end{aligned}
$$


When $=0$ and $=1$, we can write

$$
\begin{gathered}
U(\zeta ; 0)=U_{0}(\zeta), \quad U(\zeta ; 1)=U(\zeta), \\
V(\zeta ; 0)=V_{0}(\zeta), \quad V(\zeta ; 1)=V(\zeta) \\
T(\zeta ; 0)=T_{0}(\zeta), \quad T(\zeta ; 1)=T(\zeta) \\
C(\zeta ; 0)=C_{0}(\zeta), \quad C(\zeta ; 1)=C(\zeta),
\end{gathered}
$$

By Taylor's series expansion

$$
\begin{aligned}
& U(\zeta ;)=U_{0}(\zeta)+\sum_{t=1}^{\infty} U_{t}(\zeta)^{t}, \quad U_{t}=\left.\frac{1}{t !} \frac{\partial^{t} U(\zeta ;)}{\partial^{t}}\right|_{=0}, \\
& V(\zeta ;)=V_{0}(\zeta)+\sum_{t=1}^{\infty} V_{t}(\zeta)^{t}, \quad V_{t}=\left.\frac{1}{t !} \frac{\partial^{t} V(\zeta ;)}{\partial^{t}}\right|_{=0}, \\
& T\left(\zeta_{i}\right)=T_{0}(\zeta)+\sum_{t=1}^{\infty} T_{t}\left(\zeta^{t}, \quad T_{t}=\left.\frac{1}{t !} \frac{\partial^{t} T(\zeta ;)}{\partial^{t}}\right|_{=0},\right. \\
& C(\zeta ;)=C_{0}(\zeta)+\sum_{t=1}^{\infty} C_{t}(\zeta)^{t}, \quad C_{t}=\left.\frac{1}{t !} \frac{\partial^{t} C(\zeta ;)}{\partial^{t}}\right|_{=0},
\end{aligned}
$$

The auxiliary constraints are nominated such that the series (52-55) converge at $X=1$, that is,

$$
\begin{array}{r}
U(\zeta)=U_{0}(\zeta)+\sum_{t=1}^{\infty} U_{t}(\zeta) \\
V(\zeta)=V_{0}(\zeta)+\sum_{t=1}^{\infty} V_{t}(\zeta) \\
T(\zeta)=T_{0}(\zeta)+\sum_{t=1}^{\infty} T_{t}(\zeta) \\
C(\zeta)=C_{0}(\zeta)+\sum_{t=1}^{\infty} C_{t}(\zeta)
\end{array}
$$

where

$$
\chi_{t}= \begin{cases}0, & t \leq 1 \\ 1, & t>1\end{cases}
$$

Convergence of HAM

HAM certifies the series solution's convergence of the demonstrated problem. The assisting factor $\hbar$ acts a dynamic role in regulating and correcting the area of convergence of our series solution. Thus, Figure 2a,b is schemed so as to display the convergence regions for $f^{\prime}(\zeta), g(\zeta), \theta(\zeta)$, and $\phi(\zeta)$ distributions. The acceptable convergence regions of the supporting parameters $\hbar_{f}, \hbar_{g}, \hbar_{\theta}$ and $\hbar_{\phi}$ are $-2.0 \leq \hbar_{f} \leq-0.5,-2.0 \leq \hbar_{g} \leq-0.5,-2.0 \leq \hbar_{\theta} \leq 0.0$, and $-5.0 \leq \hbar_{\phi} \leq 2.0$. 


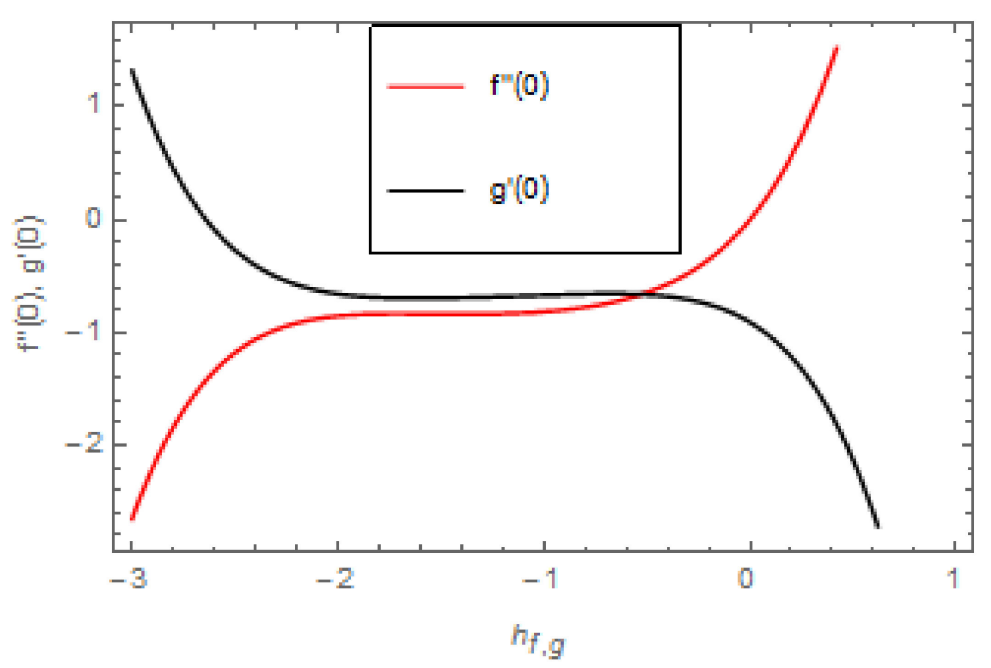

(a)

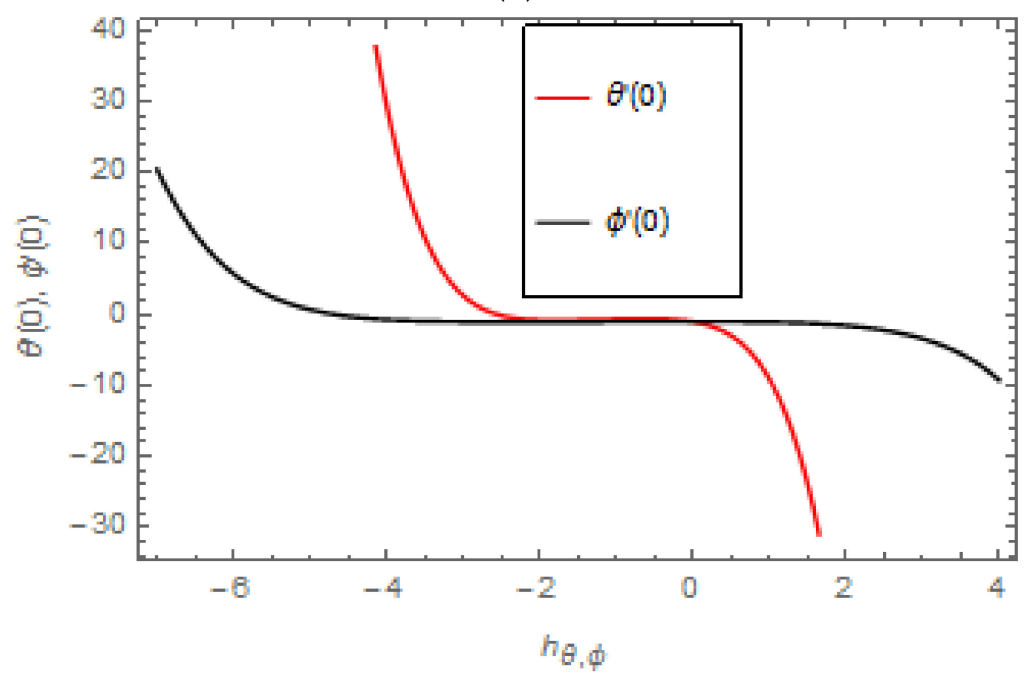

(b)

Figure 2. (a) $\hbar$ - curves for $f^{\prime \prime}(0)$ and $g^{\prime}(0) ;(\mathbf{b}) \hbar$ - curves for $\theta^{\prime}(0)$ and $\phi^{\prime}(0)$.

\section{Results and Discussion}

The coupled differential equations are treated analytically by means of HAM. Figures 3-31 are drawn with the help of computed codes in mathematica 10.0. These figures designate the variation in velocities, temperature, concentration, entropy generation, and Bejan number profiles via different indicated domains of dimensionless parameters like Casson, magnetic, Hall, mixed convection, buoyancy ratio, diffusion, chemical reaction, and radiation, and dimensionless numbers like Brinkman, Prandtl, and Schmidt. Additionally, the variation in fluid flow is studied in both circumstances of slip $(\beta \neq 0)$ and non-slip $(\beta=0)$ boundary conditions. 


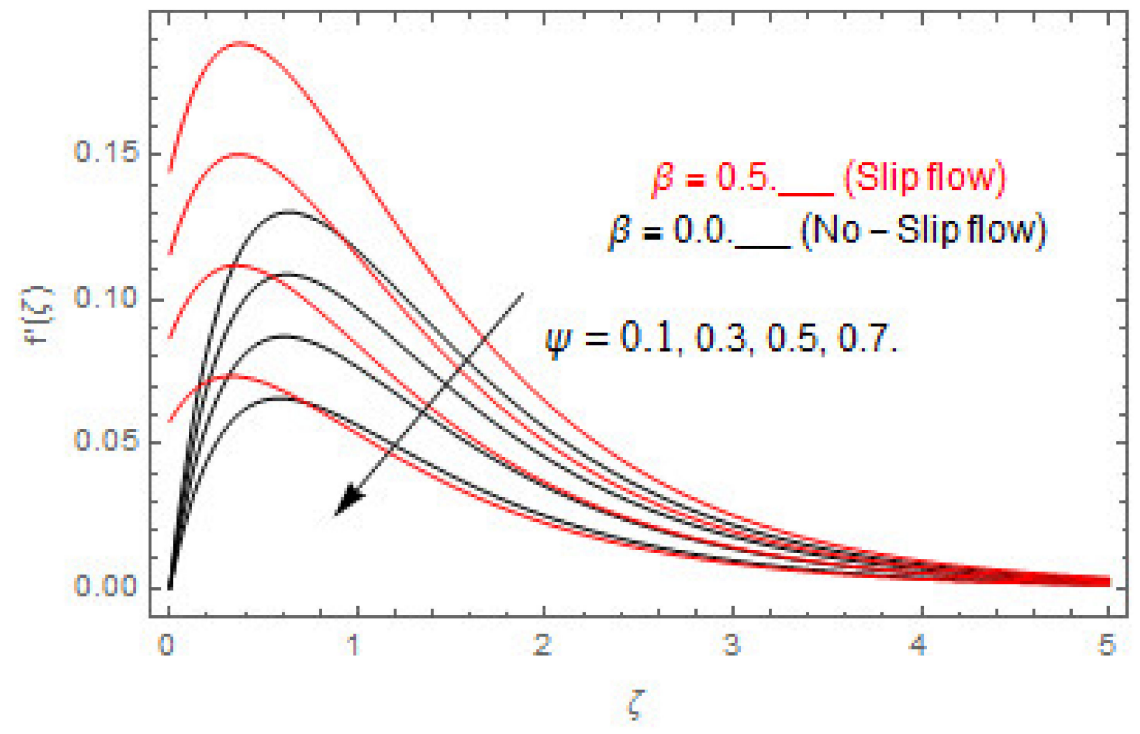

Figure 3. Via $\psi$.

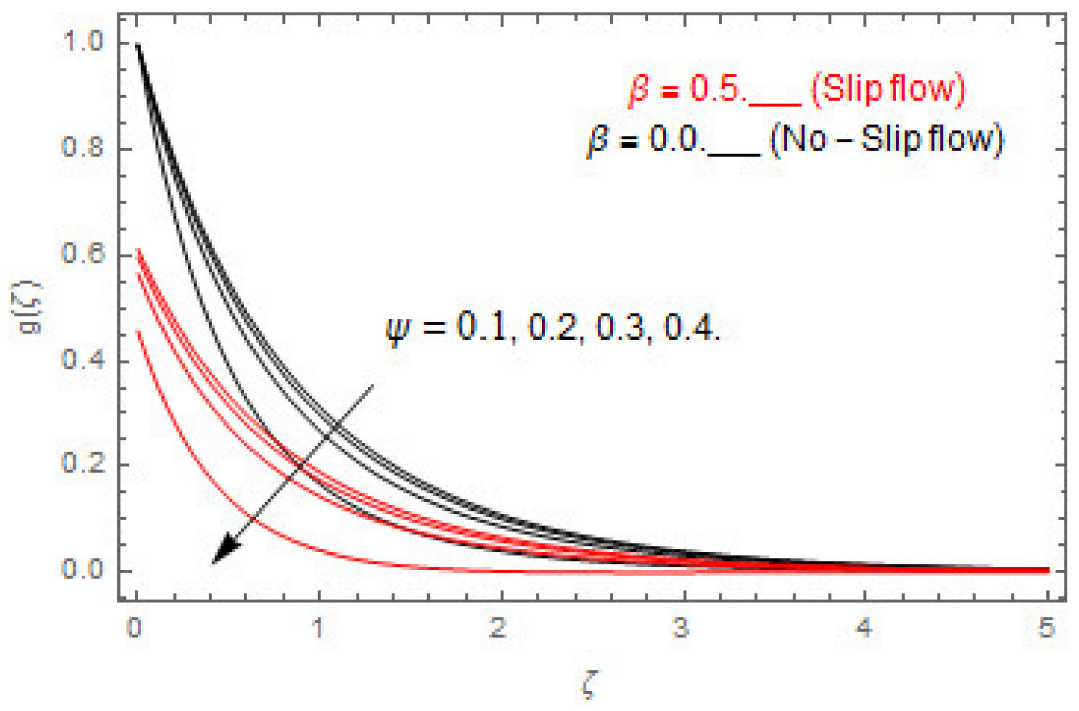

Figure 4. via $\psi$. 


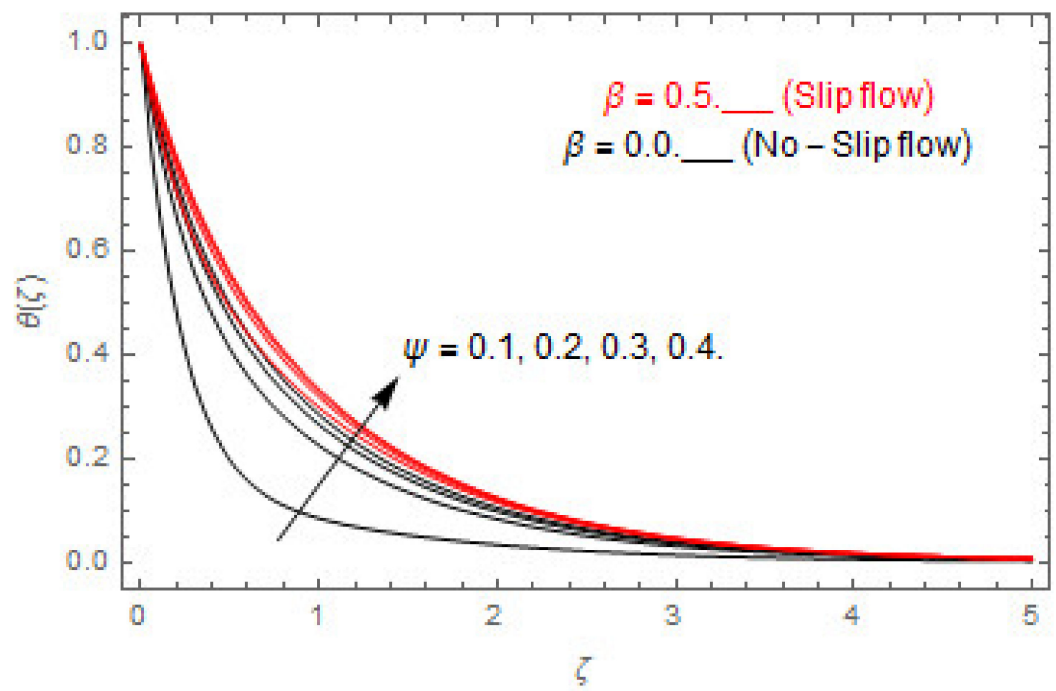

Figure 5. via $\psi$.

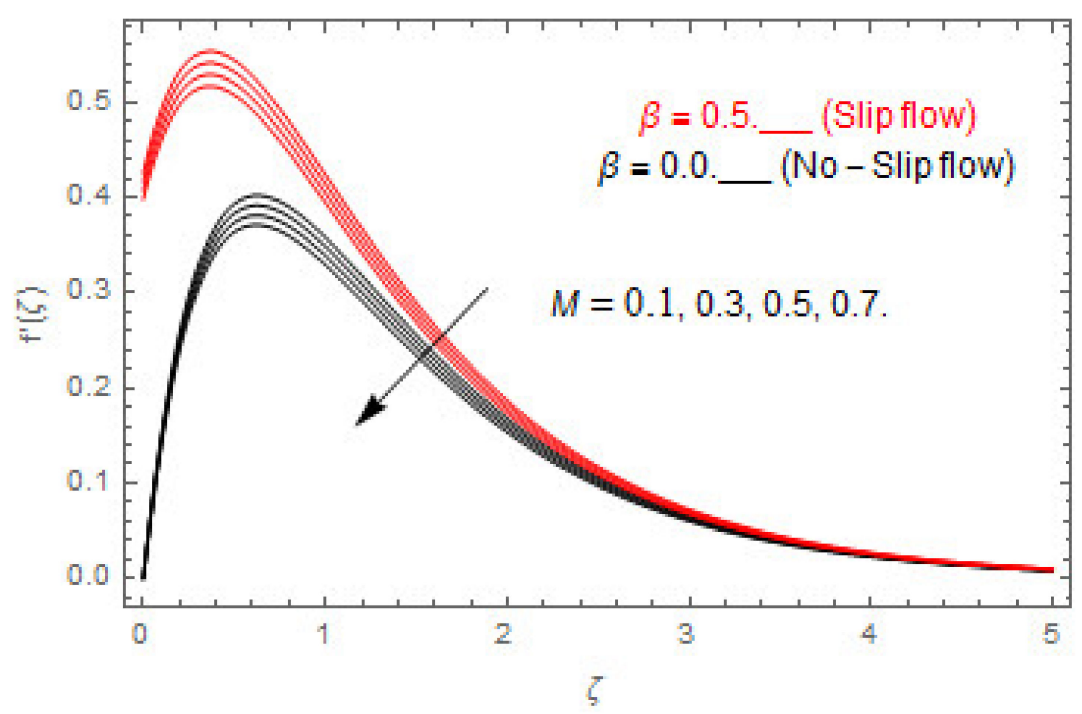

Figure 6. Via $M$.

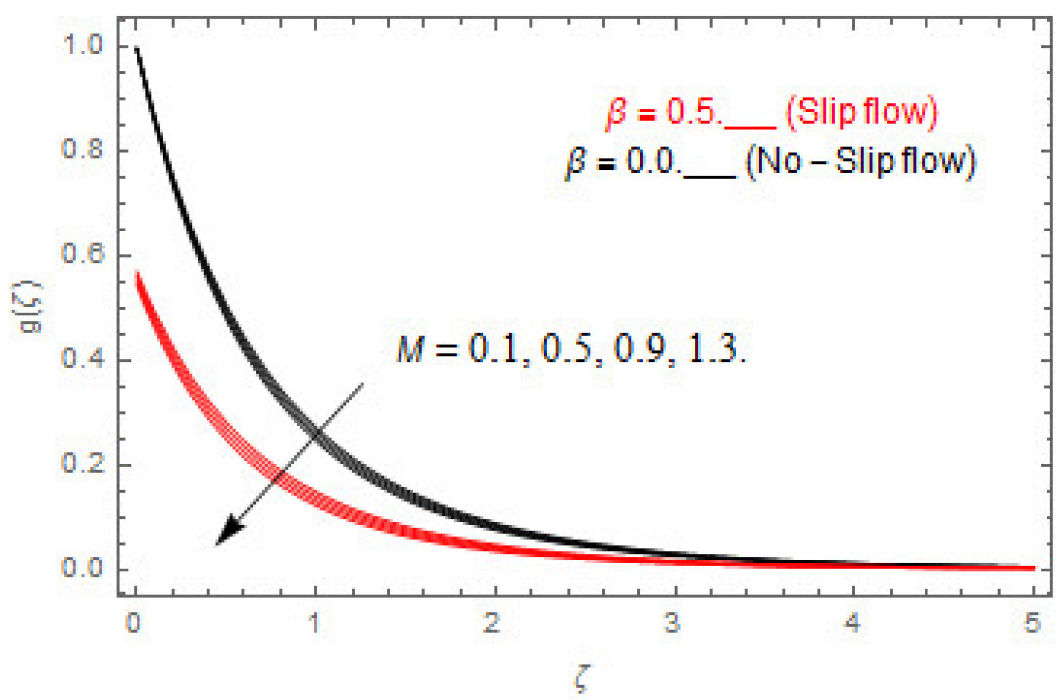

Figure 7. Via $M$. 


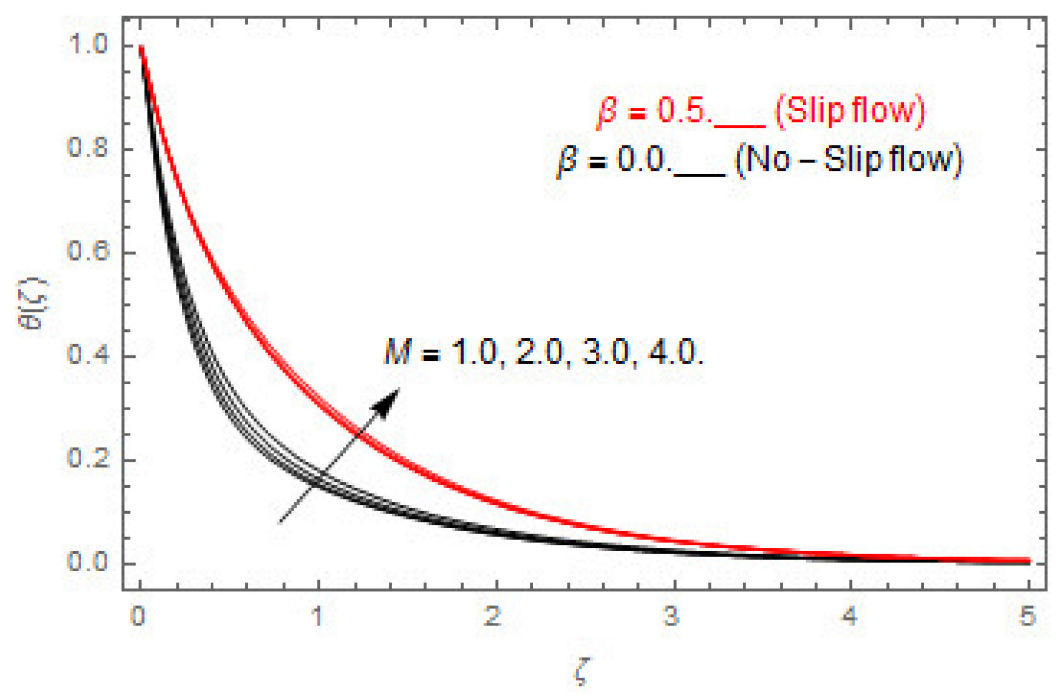

Figure 8. Via $M$.

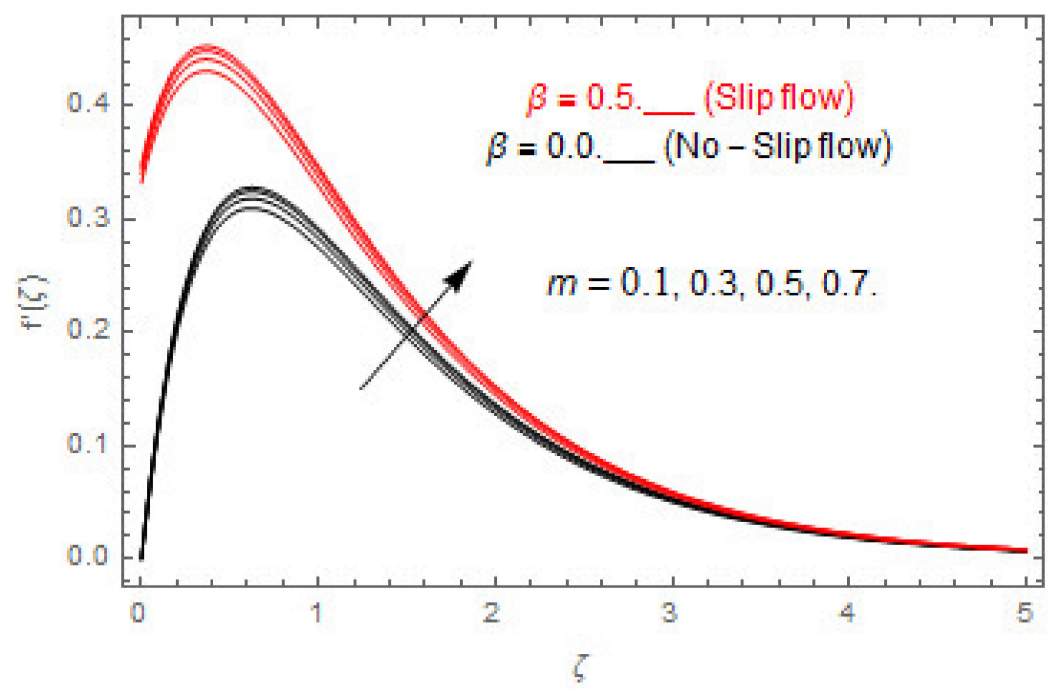

Figure 9. Via $m$.

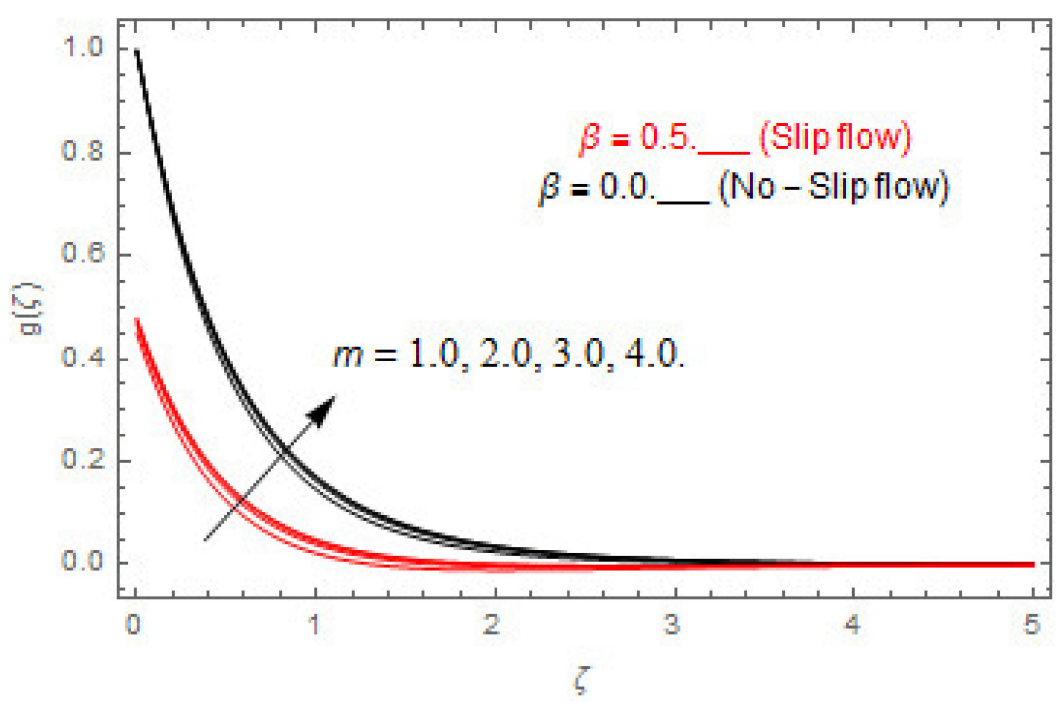

Figure 10. Via $m$. 


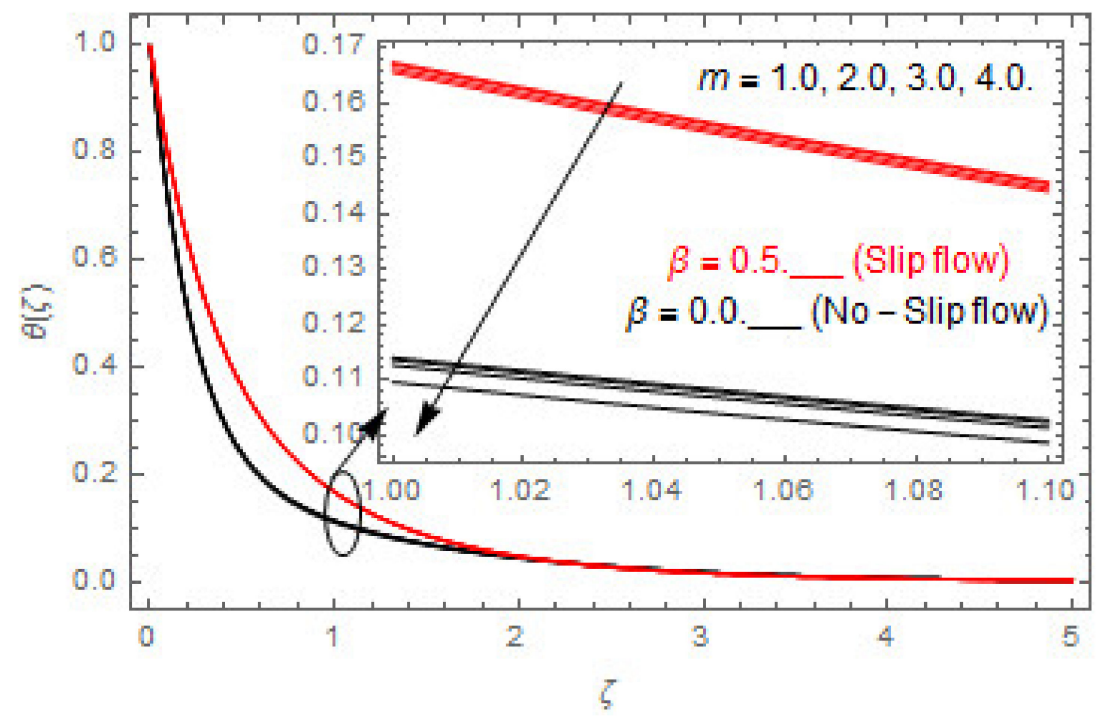

Figure 11. Via $m$.

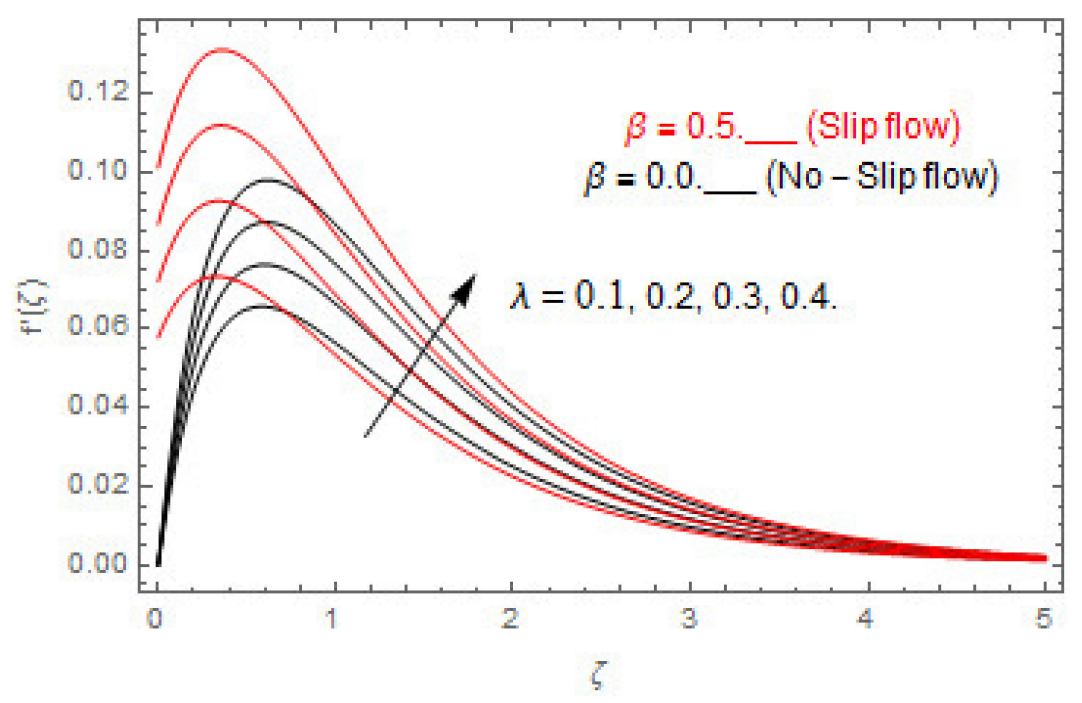

Figure 12. Via $\lambda$.

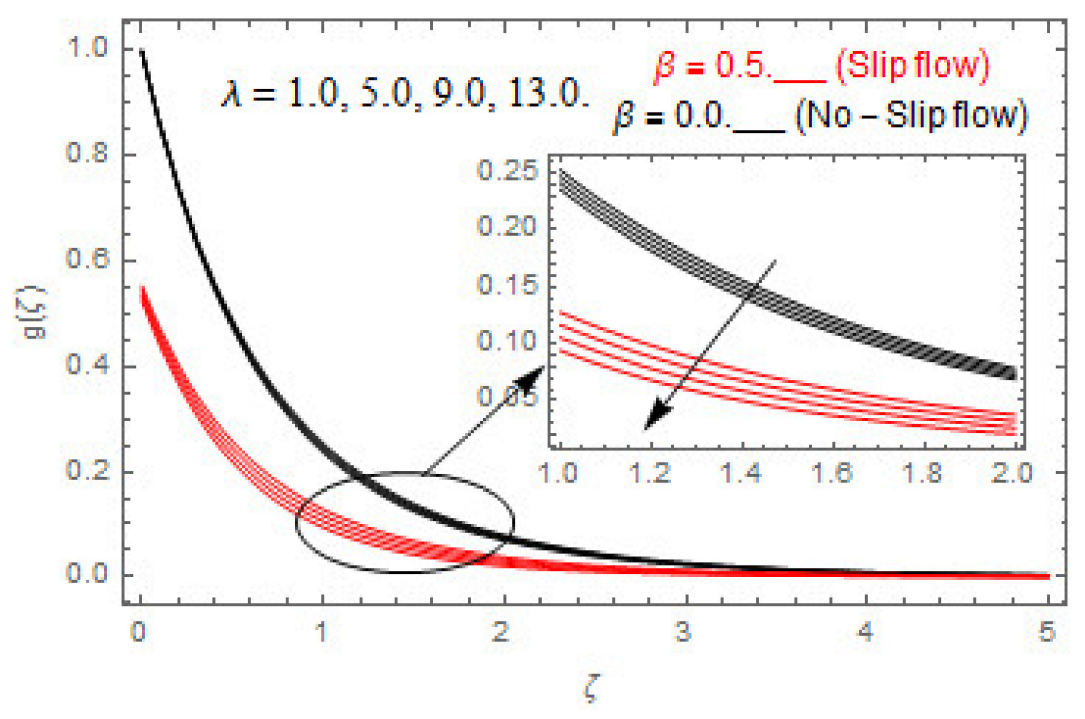

Figure 13. Via $\lambda$. 


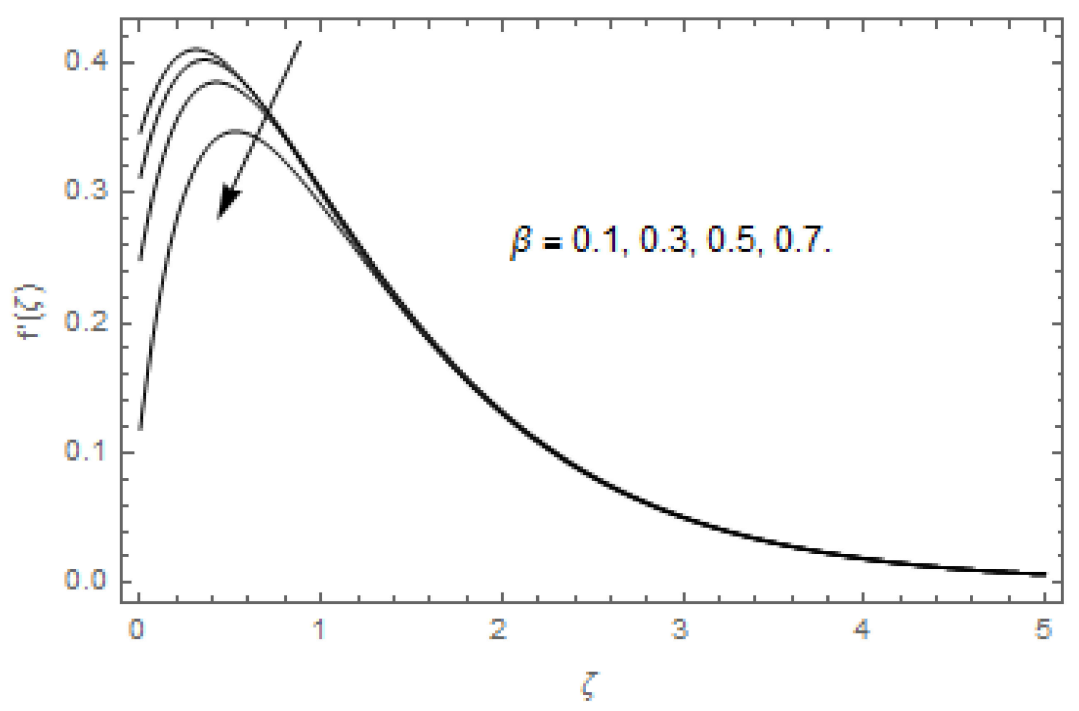

Figure 14. Via $\beta$.

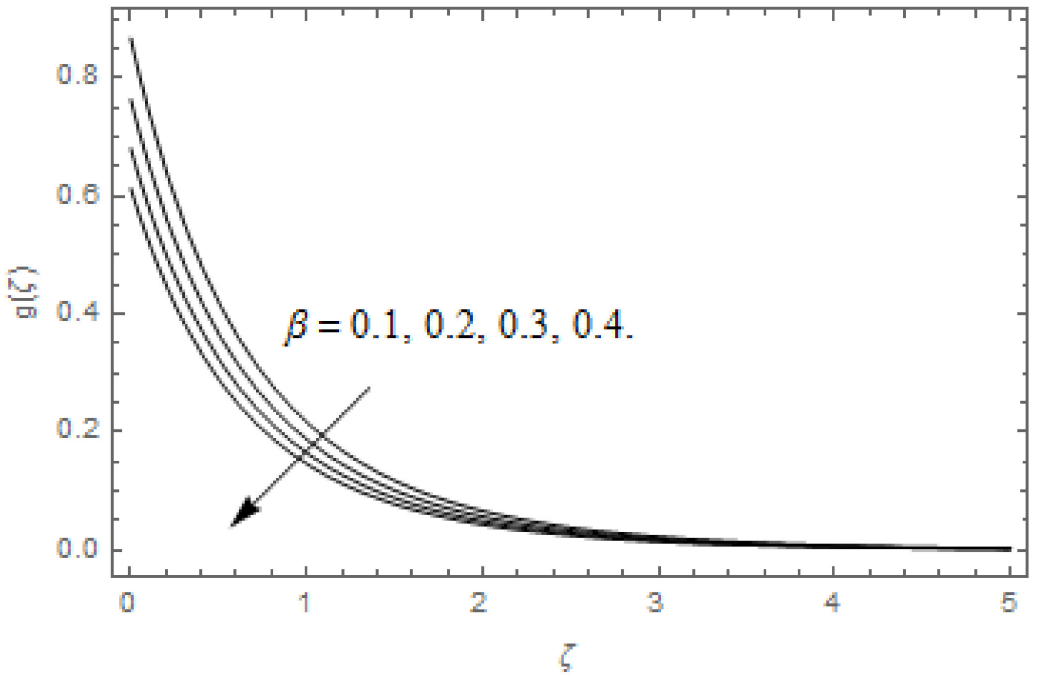

Figure 15. Via $\beta$.

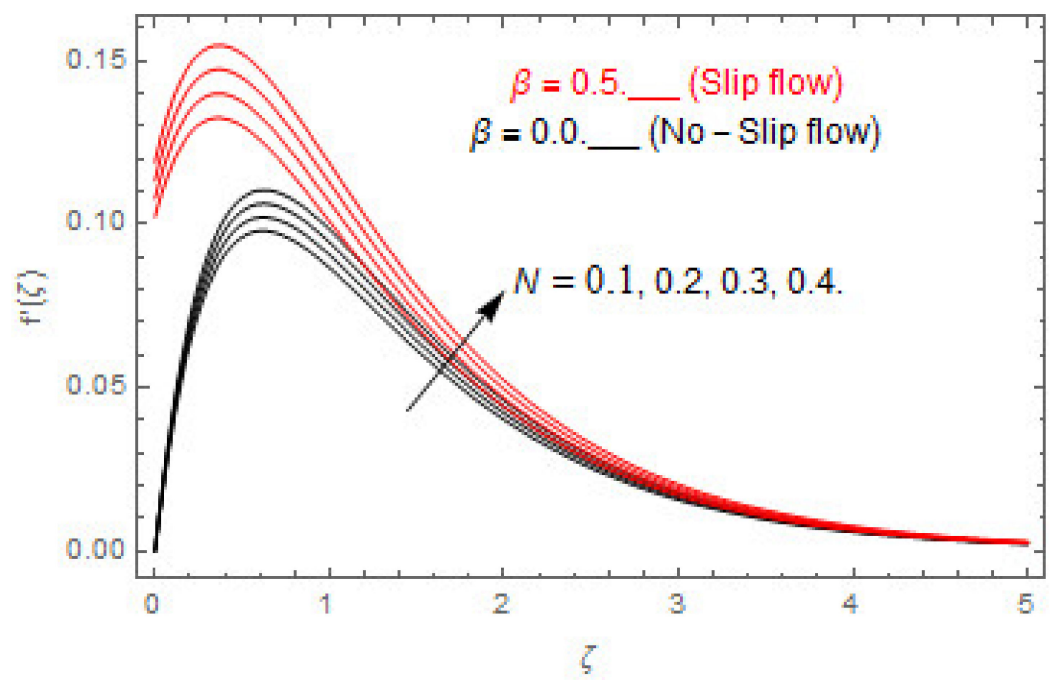

Figure 16. Via $N$. 


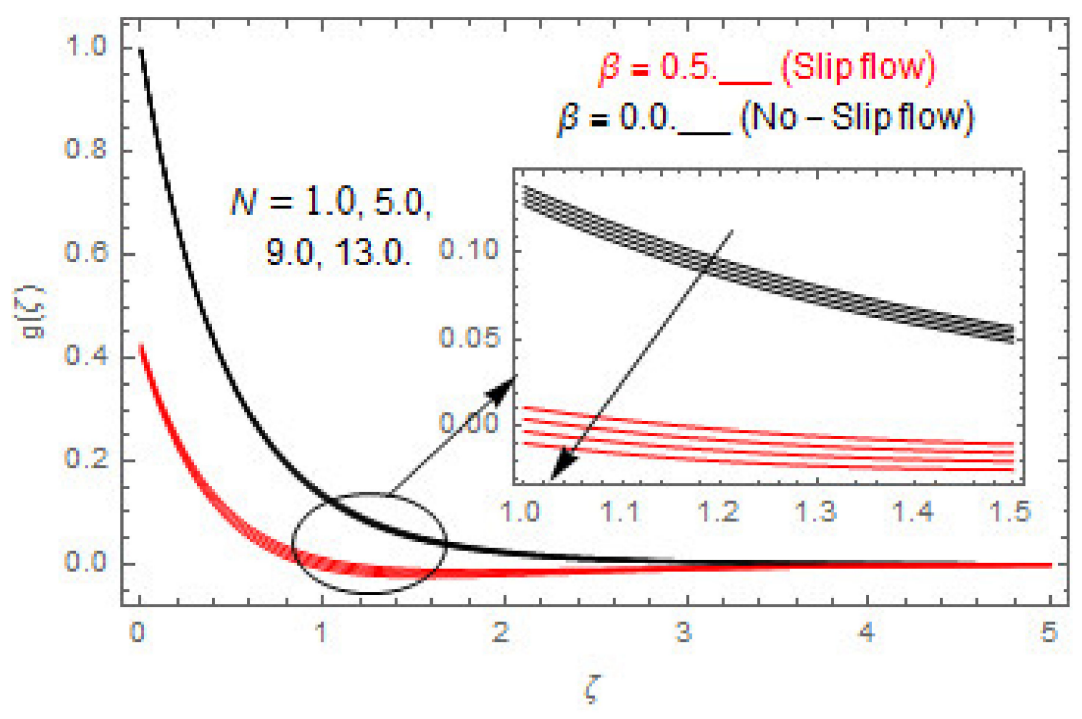

Figure 17. Via $N$.

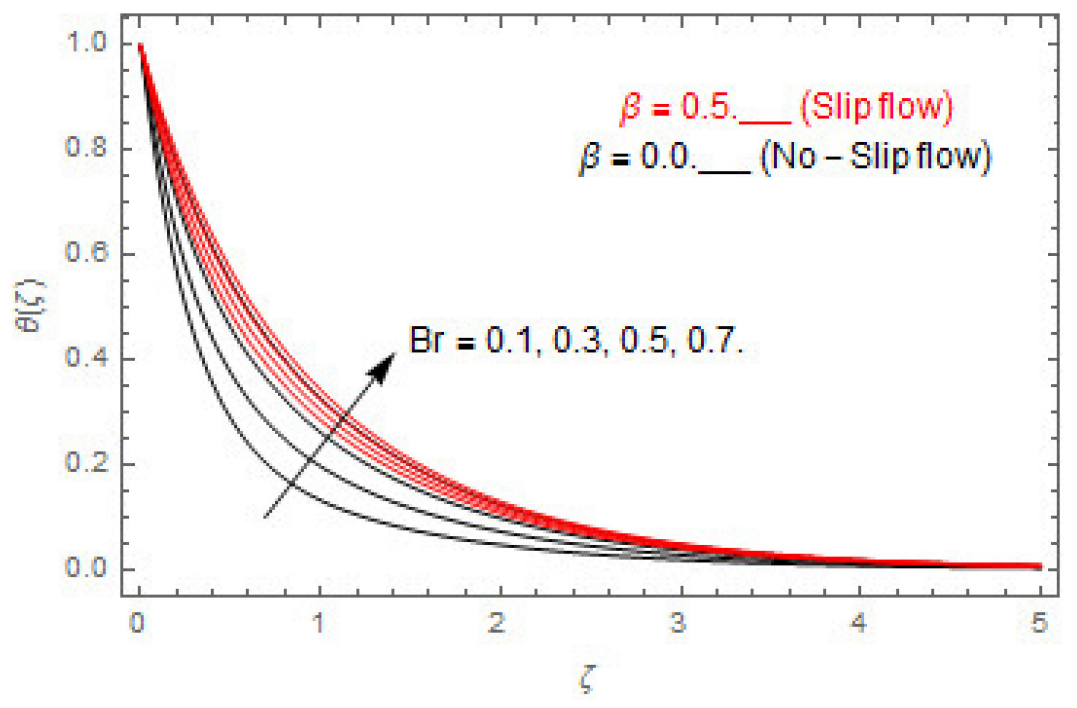

Figure 18. Via $B r$.

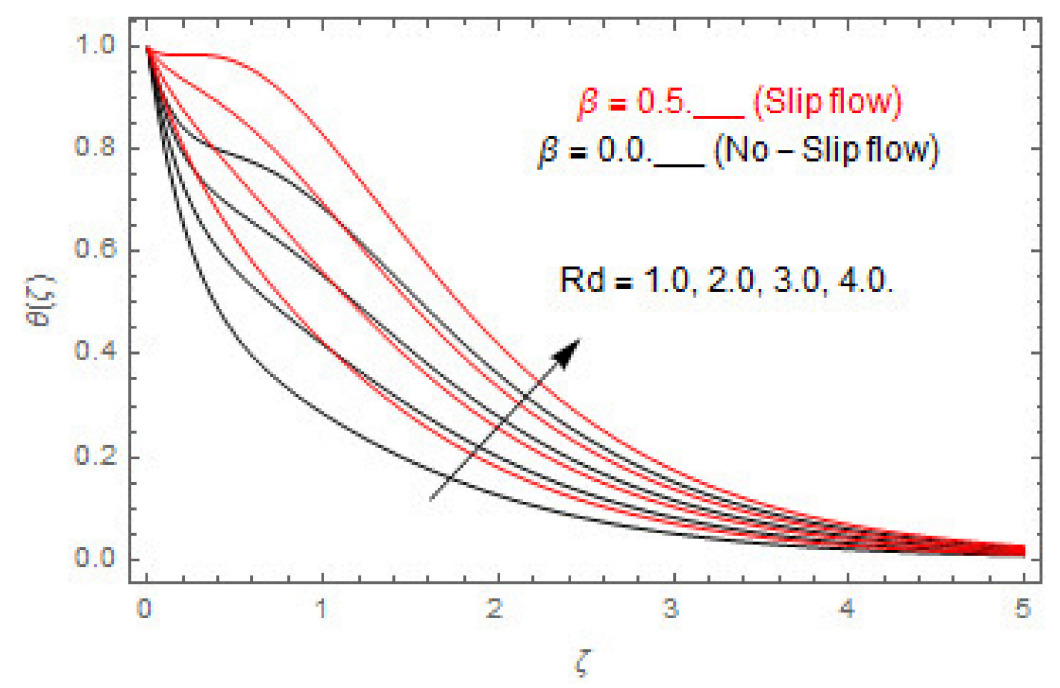

Figure 19. Via $R d$. 


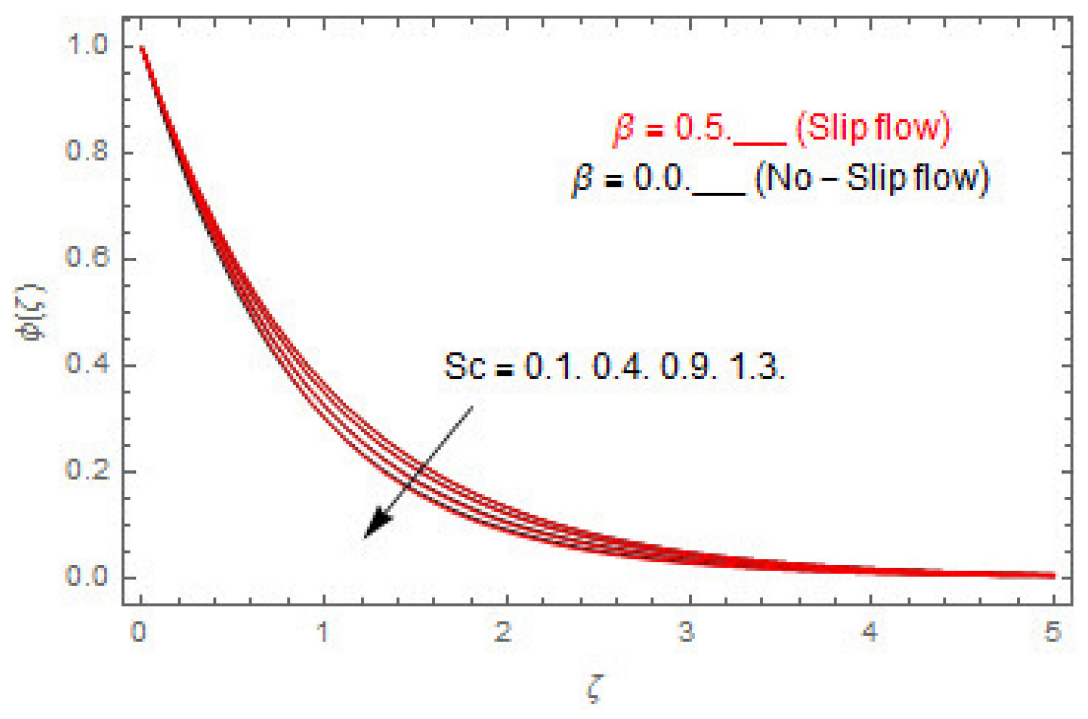

Figure 20. Via Sc.

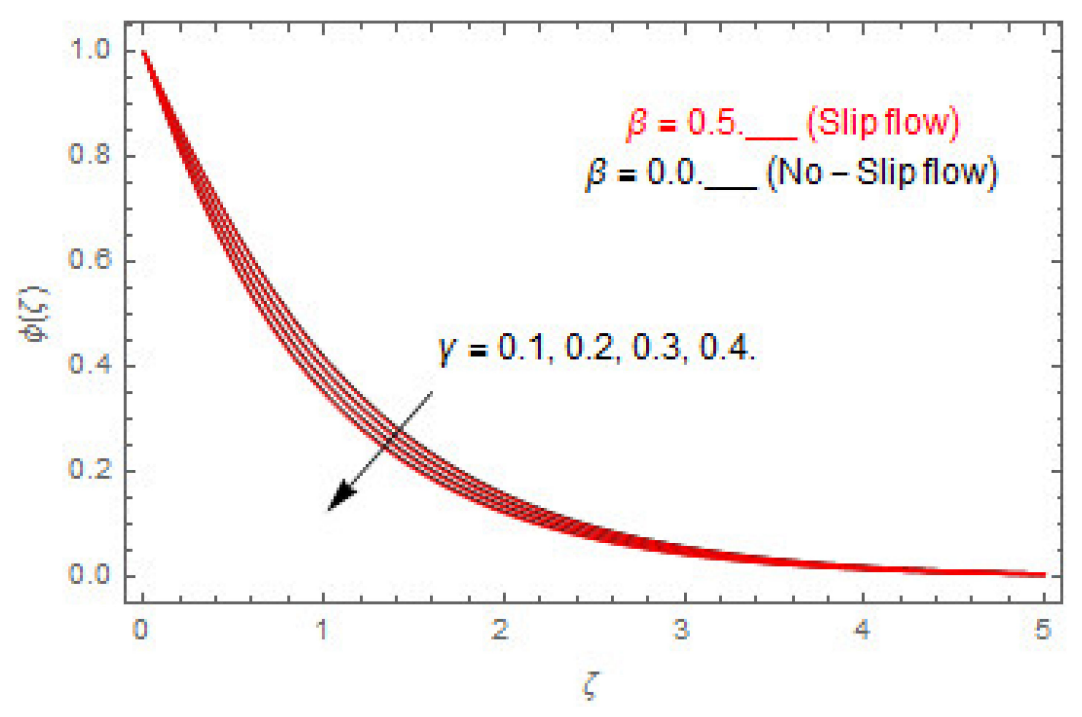

Figure 21. Via $\gamma$.

Comparisons of the present analysis with the previous analysis mentioned in the literature by Chamka et al. [10] are presented in Tables 2 and 3. The surface drag force along primary and secondary velocities components is shown in Table 2. The temperature gradient is shown in Table 3. Here, by means of present results with previous results, a great agreement is observed, which validates our present work.

Table 2. Comparison of $f^{\prime \prime}(0)$ with previous results.

\begin{tabular}{cccccc}
\hline & & \multicolumn{2}{c}{ Chamka et al. [10] } & \multicolumn{2}{c}{ Current Results } \\
\hline $\operatorname{Pr}$ & $\lambda$ & $C_{f x}$ & $\frac{1}{2} C_{f y}$ & $C_{f x}$ & $\frac{1}{2} C_{f y}$ \\
\hline 0.7 & 0.0 & 1.0255 & 0.6158 & 1.022543 & 0.615430 \\
0.7 & 1.0 & 2.2015 & 0.8494 & 2.201024 & 0.849312 \\
0.7 & 10.0 & 8.5041 & 1.3995 & 8.504256 & 1.399221 \\
10 & 0.0 & 1.0256 & 0.6158 & 1.025543 & 0.615831 \\
10 & 1.0 & 1.5636 & 0.6837 & 1.563001 & 0.683534 \\
10 & 10.0 & 2.0281 & 0.9840 & 5.082000 & 0.984555 \\
\hline
\end{tabular}




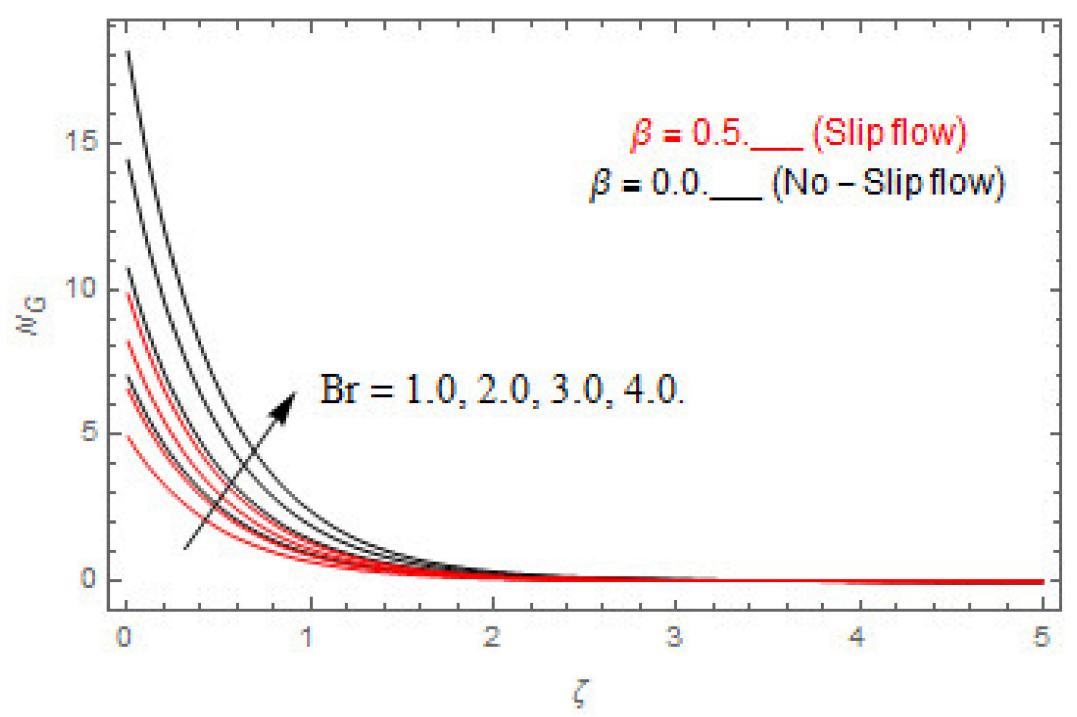

Figure 22. Via $B r$.

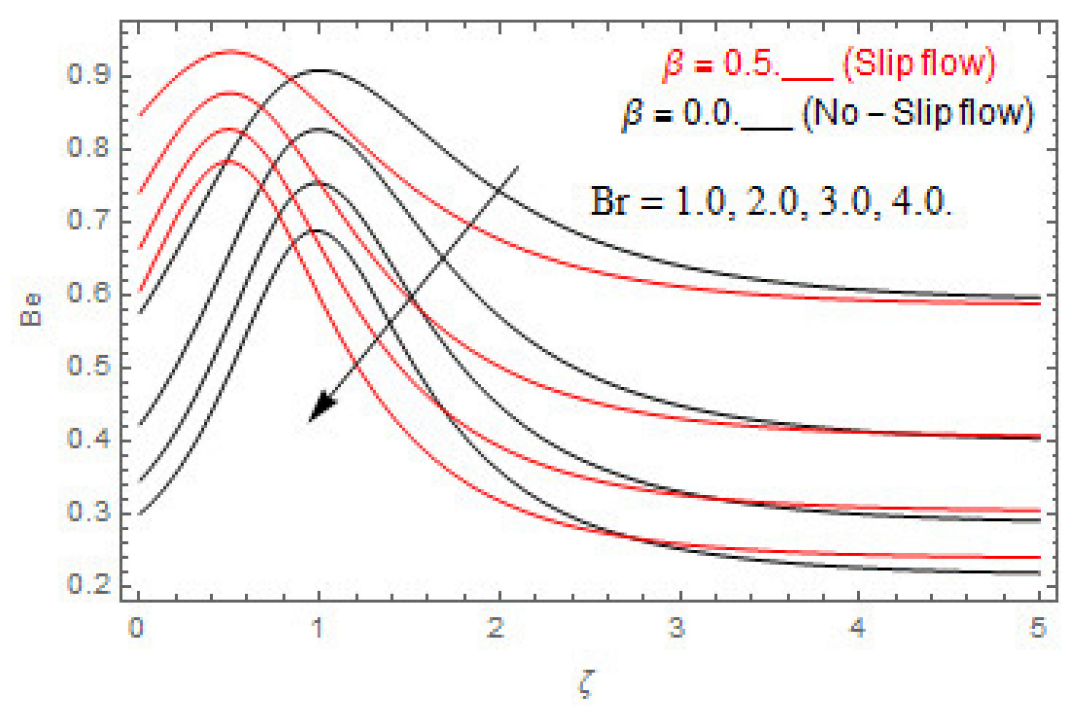

Figure 23. Via $B r$.

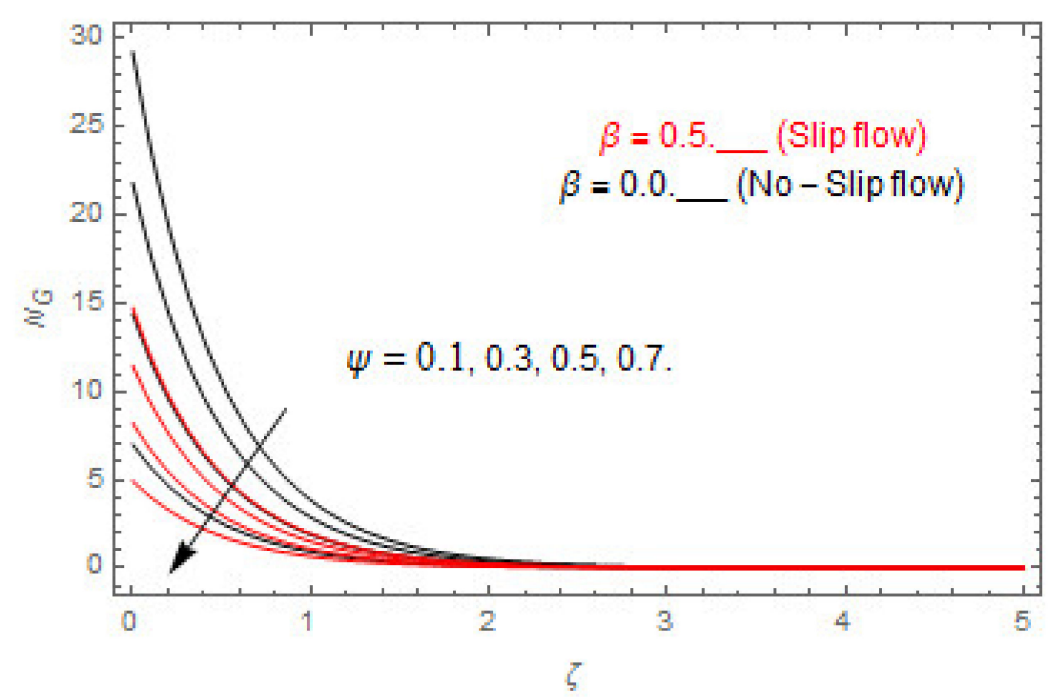

Figure 24. Via $\psi$. 


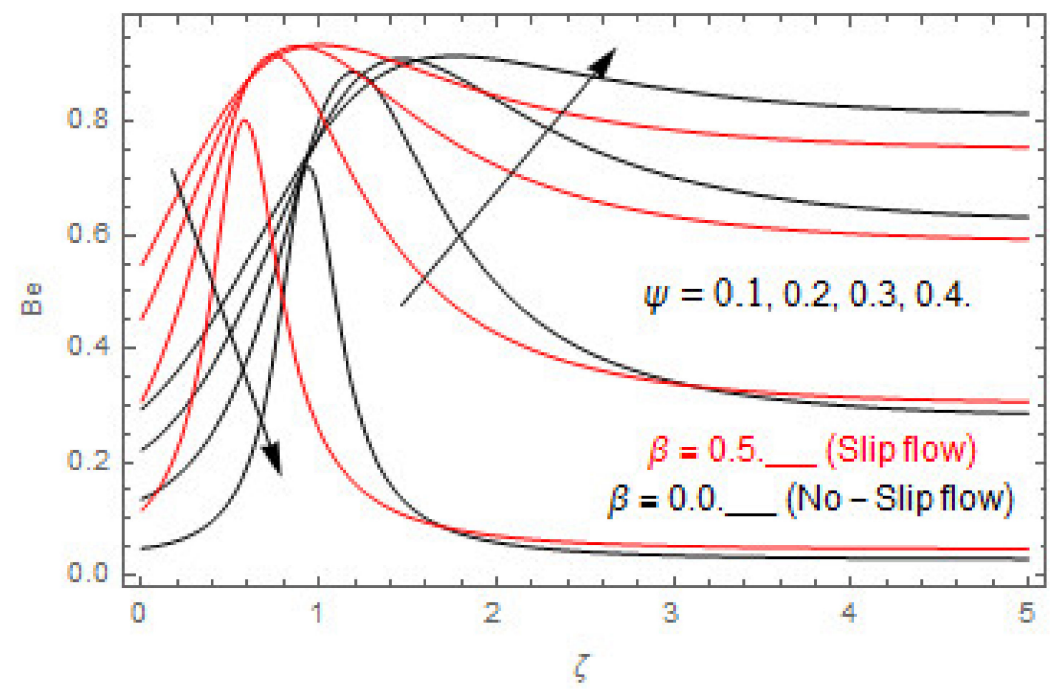

Figure 25. Via $\psi$.

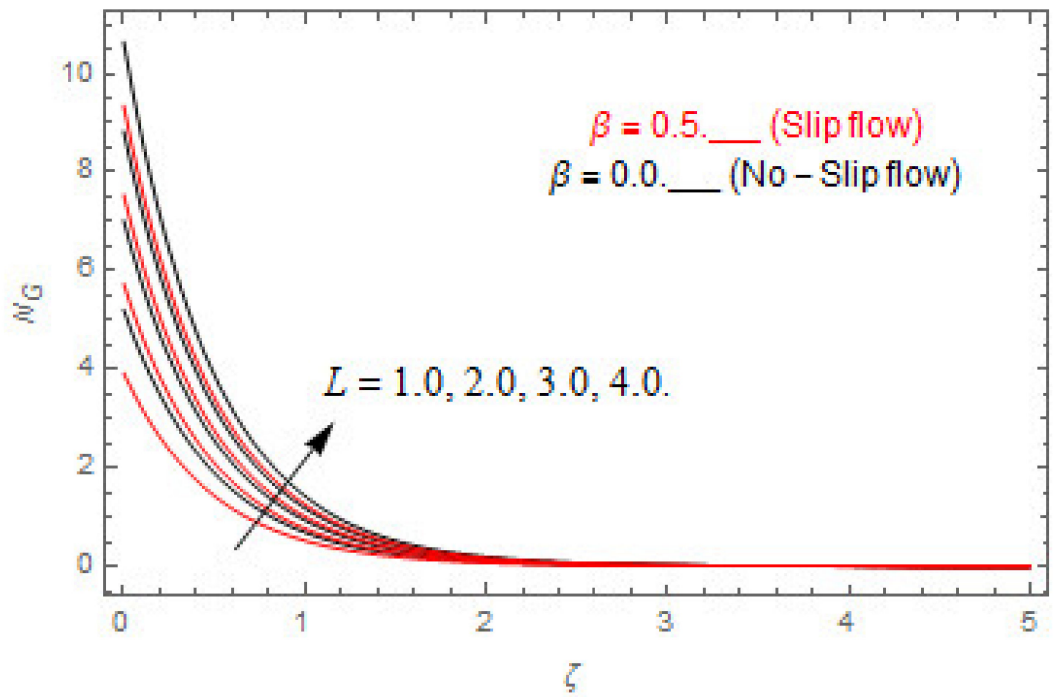

Figure 26. Via $L$.

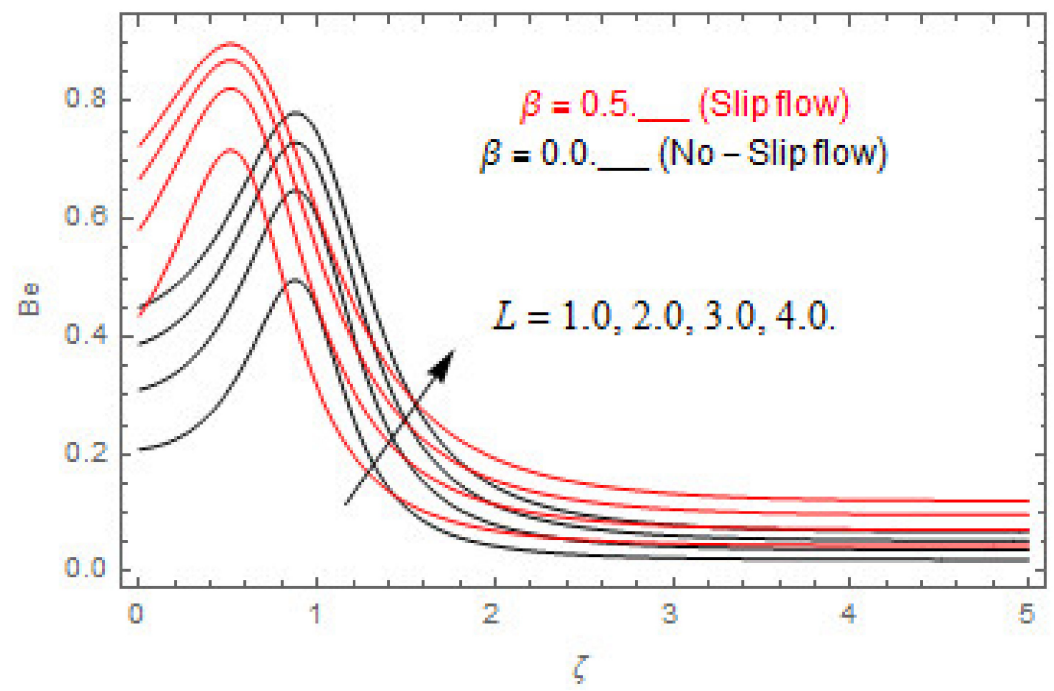

Figure 27. Via $L$. 


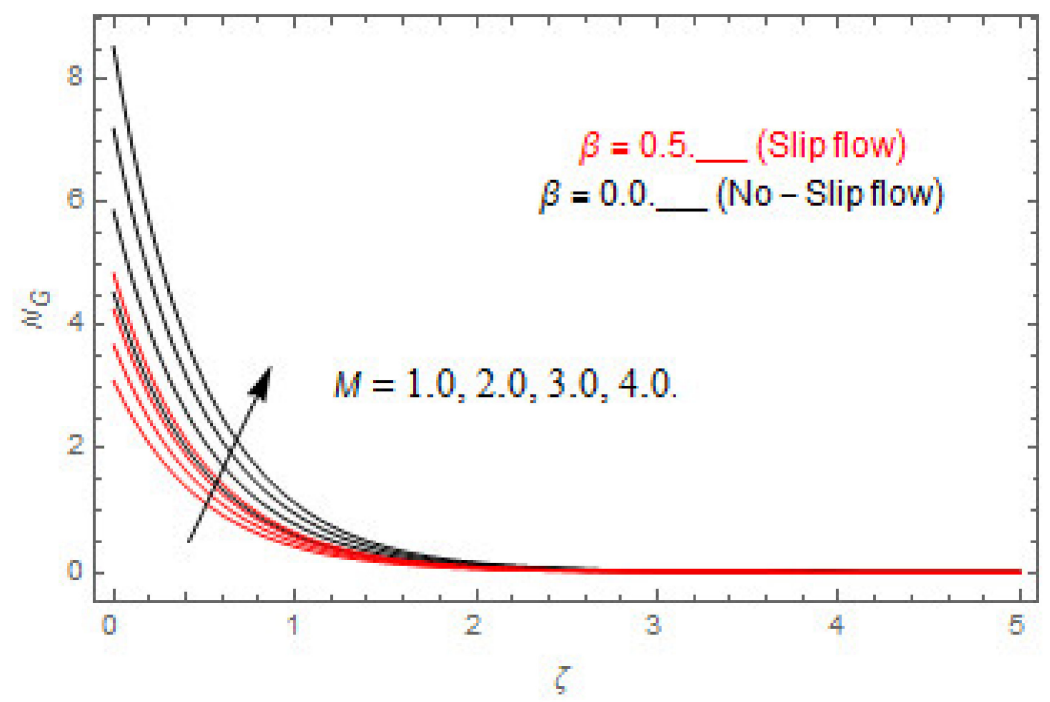

Figure 28. Via $M$.

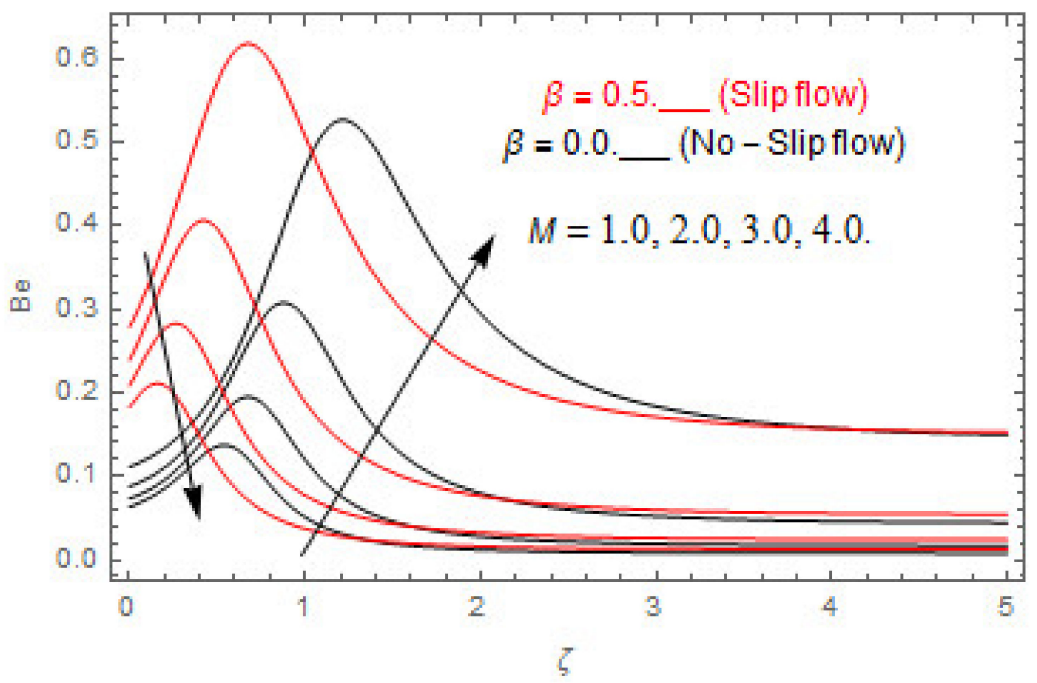

Figure 29. Via $M$.

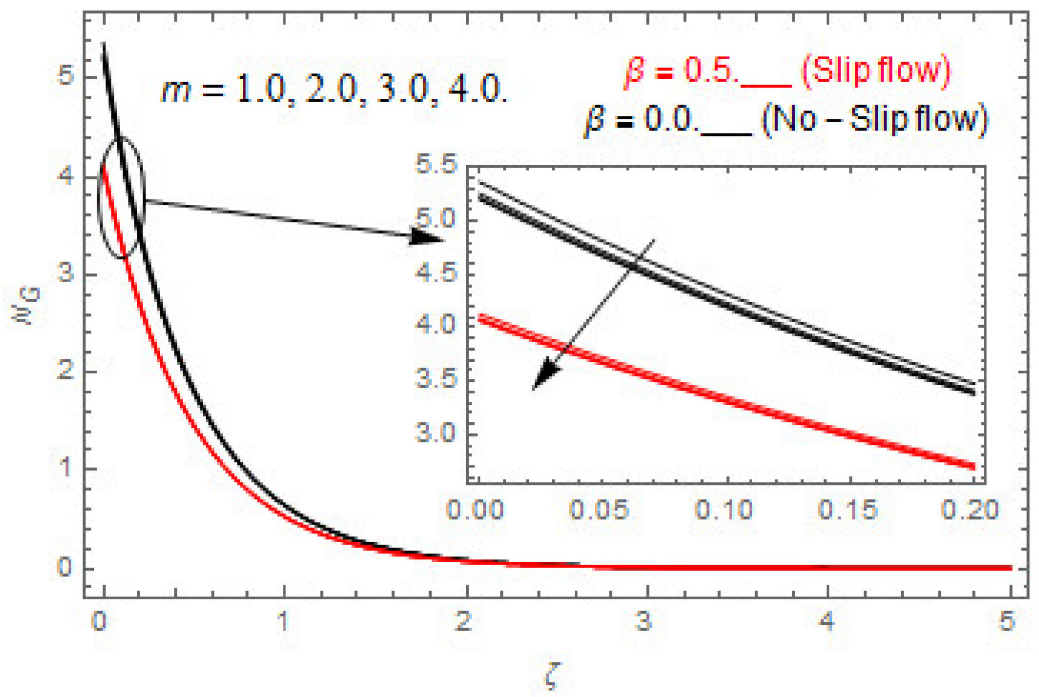

Figure 30. Via $m$. 


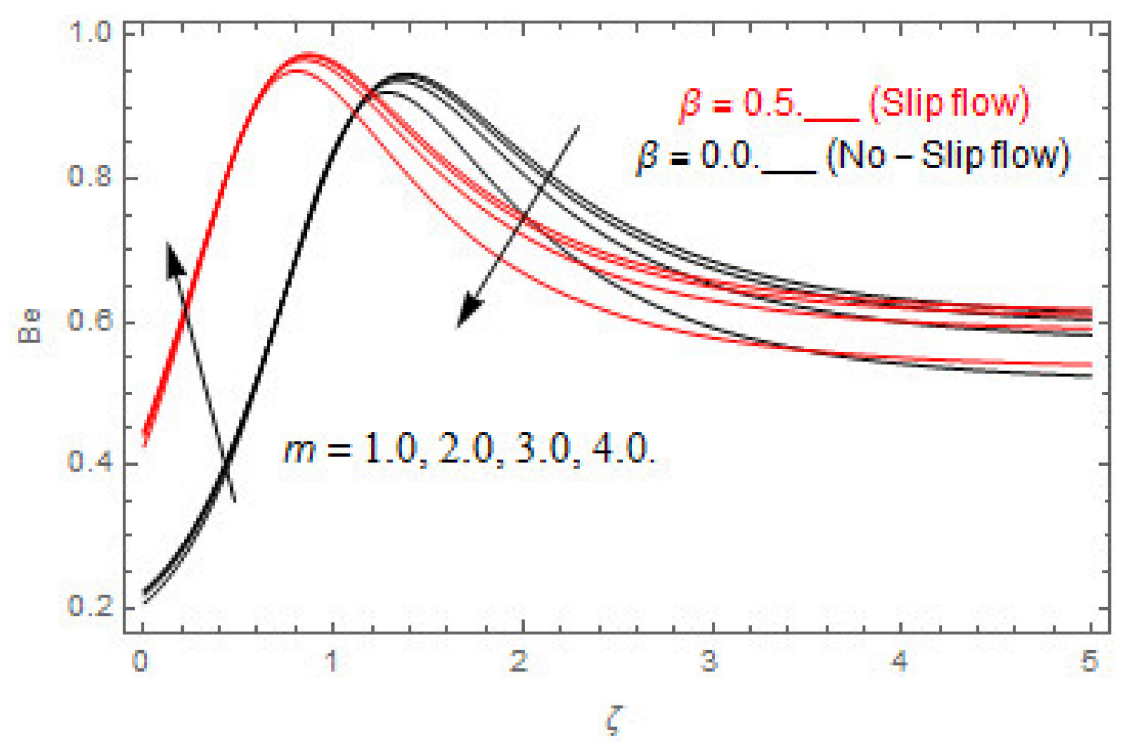

Figure 31. Via $m$.

Table 3. Comparison of $-\theta^{\prime}(0)$ with previous results.

\begin{tabular}{cccc}
\hline Pr & $\lambda$ & Chamka et al. [10] & Current Results \\
\hline 0.7 & 0.0 & 0.4299 & 0.429910 \\
0.7 & 1.0 & 0.6120 & 0.612100 \\
0.7 & 10.0 & 1.0097 & 1.399211 \\
10 & 0.0 & 1.4110 & 1.411101 \\
10 & 1.0 & 1.5662 & 1.566110 \\
10 & 10.0 & 2.3580 & 2.358102 \\
\hline
\end{tabular}

\subsection{Velocities, Temperature and Concentration Functions}

Figures 3-21 are displayed to observe the variation in velocities, temperature, and concentration functions via dimensionless parameters. Figures $3-5$ depict the variation in primary velocity, secondary velocity, and temperature profiles for both $(\beta=0, \beta=0.5)$ cases via Casson parameter $\psi$. The velocities distributions reduce with higher Casson parameter for both $(\beta=0, \beta=0.5)$ cases. Furthermore, opposite variation in temperature distribution is depicted via Casson parameter. Actually, the plastic dynamic viscosity increases with heightening in Casson parameter, which concludes in the yield stress diminishing. This phenomenon creates an opposing force to the fluid motion and escalates thermal distribution. In addition, it is obvious that the Casson parameter tends to infinity and leads to a Newtonian fluid. Figures 6-8 are displayed to depict the variation in velocities and thermal distributions via magnetic parameter. The primary and secondary velocity fields decline with swelling magnetic parameter while a contrary movement is perceived in thermal distribution. Physically, the influence of slip boundary factors and the magnetic factor create the opposing force which in result allow more liquid to pass through the surface and declines the fluid motion in both directions (i.e., $f^{\prime}(\zeta)$ and $g(\zeta)$ ). This phenomenon is owed to the fact that the Lorentz force creates resistive force to the fluid motion. In addition, the resistive force generates more heat in the fluid flow and therefore the thermal field heightens. The variation in velocities and temperature distributions via Hall parameter are published in Figures 9-11. The primary and secondary velocity distributions heighten via Hall parameter, as displayed in Figures 9 and 10. The last terms $\left(\sigma /\left(1+m^{2}\right)\right)$ displayed in Equations (7) and (8) guarantee a decline in conductivity via higher estimations of Hall parameter, which produces damping force that escalates the fluid velocity components. A descending impact of the thermal field via Hall factor is described in Figure 11. It is also depicted that the variation in non-slip flow is more than the slip flow. Figures 12 and 13 determine the variation in primary and secondary 
velocity distribution via a mixed convection parameter. The primary velocity increases via the mixed convection parameter whereas a contrasting trend is depicted for the secondary velocity of the fluid flow. Greater estimations of the mixed convection parameter escalate the fluid particles' kinetic energy, and consequently the resistive force to the particles of the fluid deescalates. Therefore, the primary velocity increases, while the secondary velocity decreases. Figures 14 and 15 illustrate the result of slip factor on primary and secondary velocity distributions. Both of the velocities distributions are reduced via higher estimations of slip parameter. This effect is palpable, as we surge the slip factor the velocity distributions of the fluid flow decline. Figures 16 and 17 display the variation in primary and secondary velocity distributions via buoyancy ratio parameter. The primary velocity distribution heightens via higher values of buoyancy ratio parameter, whereas an opposite influence is depicted against secondary velocity distribution. The variation in thermal field via Brinkman number is displayed in Figure 18. Higher estimations of Brinkman number raise the thermal field of the fluid flow. The greater estimations of Brinkman number provide a smaller amount of thermal conduction to the fluid and successively the thermal distribution heightens. Figure 19 portrays the deviation in thermal distribution via radiation parameter. Actually, the greater estimations of radiation factor produce extra heat in the fluid flow and thus the thermal distribution increases. The variation in concentration distribution via Schmidt number is displayed in Figure 20. Actually, the concentration distribution in inversely related with Schmidt number. The intensifying estimations of Schmidt number reduce the thickness of the boundary layer flow. Therefore, the concentration distribution declines. The variation in concentration distribution via chemical reaction factor is presented in Figure 21. Chemical reaction is associated with the rate of mass assignment. The local concentration deescalates with chemical reaction, and therefore the gradient of the concentration and its flux escalates. As a result, the chemical's concentration reduces with the escalation in chemical reaction parameter.

\subsection{Entropy Optimization and Bejan Number}

The deviations in entropy and Bejan number due to the dimensionless embedded parameters are displayed in Figures 22-31. Figures 22 and 23 depict the impact of Brinkman number on entropy and Bejan number, respectively. The higher estimations of Brinkman number escalate the entropy profile, while an opposite effect is observed against Bejan number. Brinkman number engenders heat in the fluid flow moving in the associated region. Thus, the entropy generation increases via Brinkman number. The opposite impact against Bejan number is observed in Figure 23. Figures 24 and 25 are displayed to observe the variation in entropy generation and Bejan number via Casson parameter, respectively. It is obvious that the entropy distribution reduces with greater estimations of Casson factor. Moreover, note that the decline in entropy profile is greater in the case of the non-slip condition compared to the slip condition. A dual impact in Bejan number via Casson parameter is depicted in Figure 25. A decreasing impact is observed via Casson parameter near the surface of the cone while opposite influence is observed after a certain space from the edge of the cone. The variation in entropy profile and Bejan number via diffusion parameter are displayed in Figures 26 and 27, respectively. Both the profiles increase with higher estimations of diffusion factor. Figures 28 and 29 are displayed to observe the variation in entropy distribution and Bejan number via magnetic parameter. Entropy distribution escalates while Bejan number de-escalates at the surface of the cone, and then escalating impact is observed away from the surface of the cone via higher values of magnetic factor. Physically, the increasing magnetic factor yields Lorentz force, which escalates the entropy optimization. The Bejan number drops with higher estimations of magnetic factor near the cone surface while opposite impact is observed after some distance from the cone surface via higher estimations of magnetic factor. Figures 30 and 31 are depicted to see the variation in entropy distribution and Bejan number via greater estimations of Hall factor. It is depicted from the figure that the heightening Hall factor reduces the entropy generation. It is obvious that Hall parameter has a direct influence on the current density and Lorentz force term. Thus, escalating Hall parameter escalates the electrical conductivity of the fluid 
which in result declines the entropy distribution. The Bejan number escalates near the cone surface where the opposite impact is observed after some distance from the surface of the cone.

\subsection{Surface Drag Force, Heat and Mass Transfer Rates}

The arithmetical values of skin friction, Nusselt, and Sherwood numbers are illustrated in Tables 4-6. As of Table 4, it is depicted that the skin friction along primary direction reduces with magnetic, Hall, buoyancy ratio, and mixed convective parameters, whereas the heightening influence is observed via Casson parameter. The surface drag force along secondary direction decreases via magnetic, Hall, and Casson parameters, whereas it increases via buoyancy ratio and mixed convection parameters. As of Table 5, the Nusselt number de-escalates with higher estimations of magnetic, thermal radiation parameters, and Brinkman number, while it escalates via Casson parameter. As of Table 6, it is perceived that the Sherwood number reduces with higher values of chemical reaction parameter and Schmidt number.

Table 4. Numerical results of skin friction along primary and secondary directions.

\begin{tabular}{ccccccc}
\hline $\boldsymbol{M}$ & $\boldsymbol{m}$ & $\boldsymbol{N}$ & $\lambda$ & $\psi$ & $\boldsymbol{C}_{f x} \sqrt{\mathbf{R e}_{x}}$ & $\frac{1}{2} \boldsymbol{C}_{f y} \sqrt{\mathbf{R e}_{x}}$ \\
\hline 0.0 & 1.0 & 0.1 & 0.1 & 0.1 & -2.52320 & 2.87840 \\
1.0 & & & & -3.29992 & 2.95818 \\
2.0 & & & -4.07658 & 3.03796 \\
& 0.1 & & & -2.80925 & 3.18393 \\
& 0.2 & & & -3.10214 & 3.17597 \\
& 0.3 & & & -3.36287 & 3.16328 \\
& & & & -3.40126 & 3.16332 \\
& 0.4 & & & -3.47804 & 3.16341 \\
& & & & -3.55481 & 3.16349 \\
& & & & -4.16530 & 3.16414 \\
& & 0.3 & & -5.38628 & 3.16478 \\
& & & & -2.28539 & 1.09543 \\
& & & 0.3 & -1.51112 & 0.67533 \\
& & & 0.4 & -1.16689 & 0.50498 \\
\hline
\end{tabular}

Table 5. Numerical results of local Nusselt number.

\begin{tabular}{ccccc}
\hline $\boldsymbol{M}$ & $\boldsymbol{R} \boldsymbol{d}$ & $\boldsymbol{B r}$ & $\boldsymbol{\psi}$ & $\boldsymbol{N} \boldsymbol{u}_{\boldsymbol{x}}$ \\
\hline 0.0 & 0.1 & 1.0 & 0.1 & 2.40758 \\
1.0 & & & 2.49147 \\
2.0 & & & 2.57265 \\
& 0.2 & & 2.82906 \\
& 0.3 & & 3.08947 \\
& 0.4 & 1.1 & & 3.35391 \\
& 1.3 & & 3.52500 \\
& 1.5 & & 3.86716 \\
& & & 0.2 & 2.20933 \\
& & & 0.3 & 2.68787 \\
& & & 0.4 & 2.40617 \\
\hline
\end{tabular}


Table 6. Numerical results of Sherwood number.

\begin{tabular}{ccc}
\hline$S c$ & $\gamma$ & $S h_{x}$ \\
\hline 0.1 & 1.0 & 1.00840 \\
0.2 & & 0.99847 \\
0.3 & 1.1 & 0.98854 \\
& 1.2 & 0.98553 \\
& 1.3 & 0.98252 \\
& & 0.97951 \\
\hline
\end{tabular}

\section{Conclusions}

An analysis of unsteady MHD Casson fluid flow with chemical reaction over a rotating cone is presented in this article. Joule heating, thermal radiation, Hall current, and viscous dissipation are considered in this work. Furthermore, the features of entropy generation with first order chemical reaction are examined. The nonlinear system of equations is tackled with HAM. The key points are enumerated underneath.

1. Primary velocity distribution reduces with heightens in Casson, velocity slip, and magnetic factors, whereas the reverse trend is observed against Hall, mixed convection, and buoyancy ratio parameters.

2. Secondary velocity distribution moderates with escalation in Casson, magnetic, mixed convection, velocity slip, and buoyancy ratio parameters, whereas the escalating impact is observed with increasing Hall parameter.

3. Temperature distribution decreases with increasing Hall parameter, whereas the rising impact is observed with escalation in Casson parameter, magnetic parameter, Brinkman number, and thermal radiation.

4. Concentration distribution decreases with increasing Schmidt number and chemical reaction parameter.

5. Entropy generation decreases with increasing Casson parameter and Hall parameter, while it increases with Brinkman number, diffusion parameter, and magnetic parameter.

6. Bejan number decreases with increasing Brinkman number and Hall parameter, whereas the increasing impact is detected with Casson parameter, diffusion parameter, and magnetic parameter.

Author Contributions: Conceptualization, methodology, software, validation, writing —original draft preparation, writing-review and editing, A.T., W.D., Z.S., and A.D.; conceptualization, methodology, software, visualization, writing—review and editing, W.D, F.A., and Z.S.; writing—review and editing, visualization, project administration, funding acquisition, investigation, resources, A.T., W.D., F.A., and A.D All authors have read and agreed to the published version of the manuscript.

Funding: This work is funded by the Deanship of Scientific Research at Majmaah University under Project Number (RGP-2019-28).

Acknowledgments: The authors extend their appreciation to the Deanship of Scientific Research at Majmaah University for funding this work under Project Number (RGP-2019-28).

Conflicts of Interest: The authors declare no conflicts of interest. 


\section{Nomenclature}

$\begin{array}{llll}\text { Angular velocity } & \Omega & \text { Gravitational acceleration } & g \\ \text { Hall parameter } & m & \text { Plastic dynamic viscosity } & \mu_{B} \\ \text { Yield stress } & S_{y} & \text { Casson parameter } & \psi \\ \text { Velocity components } & u, v, w & \text { Coordinates } & x, y, z \\ \text { Semi-vertical angle } & \alpha^{*} & \text { Velocity slip parameter } & N_{0} \\ \text { Thermal coefficient } & \beta_{t} & \text { Concentration coefficient } & \beta_{c} \\ \text { Temperature } & T & \text { Concentration } & C \\ \text { Surface temperature } & T_{w} & \text { Surface concentration } & C_{w} \\ \text { Ambient temperature } & T_{\infty} & \text { Ambient concentration } & C_{\infty} \\ \text { Stefan-Boltzman constant } & \sigma^{*} & \text { Mass diffusivity } & D_{B} \\ \text { Specific heat } & c_{p} & \text { Chemical reaction parameter } & K_{r} \\ \text { Reynolds number } & \mathrm{Re} & \text { Prandtl number } & \mathrm{Pr} \\ \text { Grashof number } & G r & \text { Eckert number } & E c \\ \text { Schmidt number } & S_{c} & \text { Brinkman } & B r \\ \text { Magnetic parameter } & M & \text { mixed convection } & \lambda \\ \text { buoyancy ratio parameter } & N & \text { Slip parameter } & \beta \\ \text { Thermal radiation parameter } & R d & \text { Dimensionless chemical reaction parameter } & \gamma \\ \text { Velocity gradients } & C_{f x}, C_{f y} & \text { Temperature gradient } & N u_{x} \\ \text { shear stresses } & \tau_{x z}, \tau_{y z} & \text { Mass gradient } & S h_{x} \\ \text { Heat flux } & q_{w} & \text { Mass flux } & h_{w} \\ \text { Entropy rate } & N_{G} & \text { Diffusion parameter } & L \\ \text { Temperature difference } & T_{d} & \text { Concentration difference } & C_{d} \\ \text { Dimensionless parameter } & A & & \end{array}$

\section{References}

1. Kumari, M.; Pop, I.; Nath, G. Mixed convection along a vertical cone. Int. Commun. Heat Mass Transf. 1989, 16, 247-255. [CrossRef]

2. Nadeem, S.; Saleem, S. Analytical treatment of unsteady mixed convection MHD flow on a rotating cone in a rotating frame. J. Taiwan Inst. Chem. Eng. 2013, 44, 596-604. [CrossRef]

3. Hayat, T.; Khan, S.A.; Khan, M.I.; Alsaedi, A. Irreversibility characterization and investigation of mixed convective reactive flow over a rotating cone. Comput. Methods Programs Biomed. 2020, 185, 105168. [CrossRef] [PubMed]

4. Sulochana, C.; Samrat, S.; Sandeep, N. Numerical investigation of magnetohydrodynamic (MHD) radiative flow over a rotating cone in the presence of Soret and chemical reaction. Propuls. Power Res. 2018, 7, 91-101. [CrossRef]

5. Raju, C.S.K.; Sandeep, N. Heat and mass transfer in MHD non-Newtonian bio-convection flow over a rotating cone/plate with cross diffusion. J. Mol. Liq. 2016, 215, 115-126. [CrossRef]

6. Zohra, F.; Uddin, M.J.; Ismail, A.; Bég, O.A.; Kadir, A. Anisotropic slip magneto-bioconvection flow from a rotating cone to a nanofluid with Stefan blowing effects. Chin. J. Phys. 2018, 56, 432-448. [CrossRef]

7. Raju, C.S.K.; Sandeep, N. Unsteady Casson nanofluid flow over a rotating cone in a rotating frame filled with ferrous nanoparticles: A numerical study. J. Magn. Magn. Mater. 2017, 421, 216-224. [CrossRef]

8. Nadeem, S.; Saleem, S. Analytical Study of Rotating Non-Newtonian Nanofluid on a Rotating Cone. J. Thermophys. Heat Transf. 2014, 28, 295-302. [CrossRef]

9. Ravindran, R.; Roy, S.J.; Momoniat, E. Effects of injection (suction) on a steady mixed convection boundary layer flow over a vertical cone. Int. J. Numer. Methods Heat Fluid Flow 2009, 19, 432-444. [CrossRef]

10. Chamkha, A.J.; Al-Mudhaf, A. Unsteady heat and mass transfer from a rotating vertical cone with a magnetic field and heat generation or absorption effects. Int. J. Therm. Sci. 2005, 44, 267-276. [CrossRef]

11. Sato, H. The Hall Effect in the Viscous Flow of Ionized Gas Between Two Parallel Plates under Transverse Magnetic Field. J. Phys. Soc. Jpn. 1961, 16, 1427-1433. [CrossRef]

12. Sherman, A.; Sutton, G.W. Magnetohydrodynamics; Northwestern University Press: Evanston, IL, USA, 1962. 
13. Katagiri, M. The Effect of Hall Currents on the Magnetohydrodynamic Boundary Layer Flow past a Semi-Infinite Flate Plate. J. Phys. Soc. Jpn. 1969, 27, 1051-1059. [CrossRef]

14. Pop, I.; Soundalgekar, V.M. Effects of Hall current on hydromagnetic flow near a porous plate. Acta Mech. 1974, 20, 315-318. [CrossRef]

15. Gupta, A.S. Hydromagnetic flow past a porous flat plate with hall effects. Acta Mech. 1975, 22, $281-287$. [CrossRef]

16. Jana, R.N.; Gupta, A.S.; Datta, N. Hall Effects on the Hydromagnetic Flow past an Infinite Porous Flat Plate. J. Phys. Soc. Jpn. 1977, 43, 1767-1772. [CrossRef]

17. Pop, I.; Watanabe, T. Hall effects on magnetohydrodynamic free convection about a semi-infinite vertical flat plate. Int. J. Eng. Sci. 1994, 32, 1903-1911. [CrossRef]

18. El-Aziz, M.A. Flow and heat transfer over an unsteady stretching surface with Hall effect. Meccanica 2009, 45, 97-109. [CrossRef]

19. Seth, G.S.; Singh, J. Mixed convection hydromagnetic flow in a rotating channel with Hall and wall conductance effects. Appl. Math. Model. 2016, 40, 2783-2803. [CrossRef]

20. Abdel-Wahed, M.S. Magnetohydrodynamic Ferro-Nano fluid flow in a semi-porous curved tube under the effect of hall current and nonlinear thermal radiative. J. Magn. Magn. Mater. 2019, 474, 347-354. [CrossRef]

21. Osalusi, E.; Side, J.; Harris, R.; Clark, P. The effect of combined viscous dissipation and Joule heating on unsteady mixed convection MHD flow on a rotating cone in a rotating fluid with variable properties in the presence of Hall and ion-slip currents. Int. Commun. Heat Mass Transf. 2008, 35, 413-429. [CrossRef]

22. Casson, N. A Flow Equation for Pigment-Oil Suspensions of the Printing Ink Type. In Rheology of Disperse Systems; Mill, C.C., Ed.; Pergamon: New York, NY, USA, 1959.

23. Hayat, T.; Shehzad, S.A.; Alsaedi, A.; Alhothuali, M.S. Mixed Convection Stagnation Point Flow of Casson Fluid with Convective Boundary Conditions. Chin. Phys. Lett. 2012, 29, 114704. [CrossRef]

24. Eldabe, N.T.M.; Salwa, M.G.E. Heat transfer of MHD Non-Newtonian Casson Fluid Flow between two Rotating Cylinders. J. Phys. Soc. Jpn. 1995, 64, 41-64.

25. Malik, M.Y.; Naseer, M.; Nadeem, S.; Rehman, A. The boundary layer flow of Casson nanofluid over a vertical exponentially stretching cylinder. Appl. Nanosci. 2013, 4, 869-873. [CrossRef]

26. El-Aziz, M.A.; Afify, A.A. Effects of Variable Thermal Conductivity with Thermal Radiation on MHD Flow and Heat Transfer of Casson Liquid Film Over an Unsteady Stretching Surface. Braz. J. Phys. 2016, 46, 516-525. [CrossRef]

27. Shateyi, S.; Mabood, F.; Lorenzini, G. Casson fluid flow: Free convective heat and mass transfer over an unsteady permeable stretching surface considering viscous dissipation. J. Eng. Thermophys. 2017, 26, 39-52. [CrossRef]

28. Shehzad, S.A.; Hayat, T.; Alsaedi, A. MHD flow of a Casson fluid with power law heat flux and heat source. Comput. Appl. Math. 2017, 37, 2932-2942. [CrossRef]

29. Reddy, J.R.; Sugunamma, V.; Sandeep, N. Enhanced heat transfer in the flow of dissipative non-Newtonian Casson fluid flow over a convectively heated upper surface of a paraboloid of revolution. J. Mol. Liq. 2017, 229, 380-388. [CrossRef]

30. Bejan, A. A Study of Entropy Generation in Fundamental Convective Heat Transfer. J. Heat Transf. 1979, 101, 718-725. [CrossRef]

31. Bejan, A. Second-Law Analysis in Heat Transfer and Thermal Design. Advances in Heat Transfer 1982, 15, 1-58. [CrossRef]

32. Makinde, O.D. Thermodynamic second law analysis for a gravity-driven variable viscosity liquid film along an inclined heated plate with convective cooling. J. Mech. Sci. Technol. 2010, 24, 899-908. [CrossRef]

33. Marzougui, S.; Mebarek-Oudina, F.; Assia, A.; Magherbi, M.; Shah, Z.; Ramesh, K. Entropy generation on magneto-convective flow of copper-water nanofluid in a cavity with chamfers. J. Therm. Anal. Calorim. 2020, 1-12. [CrossRef]

34. Tshehla, M.S.; Makinde, O.D. Analysis of Entropy Generation in a Variable Viscosity Fluid Flow Between Two Concentric Pipes with Convective Cooling at the Surface. Int. J. Phys. Sci. 2011, 6, 6053-6060.

35. Chen, C.-K.; Lai, H.-Y.; Liu, C.-C. Numerical analysis of entropy generation in mixed convection flow with viscous dissipation effects in vertical channel. Int. Commun. Heat Mass Transf. 2011, 38, 285-290. [CrossRef]

36. Liu, C.-C.; Lo, C.-Y. Numerical analysis of entropy generation in mixed-convection MHD flow in vertical channel. Int. Commun. Heat Mass Transf. 2012, 39, 1354-1359. [CrossRef] 
37. Rashidi, M.M.; Abelman, S.; Mehr, N.F. Entropy generation in steady MHD flow due to a rotating porous disk in a nanofluid. Int. J. Heat Mass Transf. 2013, 62, 515-525. [CrossRef]

38. Shah, Z.; Kumam, P.; Deebani, W. Radiative MHD Casson Nanofluid Flow with Activation energy and chemical reaction over past nonlinearly stretching surface through Entropy generation. Sci. Rep. 2020, 10, 4402-4414. [CrossRef]

39. Shah, Z.; Alzahrani, E.O.; Dawar, A.; Alghamdi, W.; Ullah, M.Z. Entropy Generation in MHD Second-Grade Nanofluid Thin Film Flow Containing CNTs with Cattaneo-Christov Heat Flux Model past an Unsteady Stretching Sheet. Appl. Sci. 2020, 10, 2720. [CrossRef]

40. Qing, J.; Bhatti, M.; Abbas, M.A.; Rashidi, M.M.; Ali, M. Entropy Generation on MHD Casson Nanofluid Flow over a Porous Stretching/Shrinking Surface. Entropy 2016, 18, 123. [CrossRef]

41. Reddy, G.J.; Kethireddy, B.; Kumar, M.; Hoque, M.M. A molecular dynamics study on transient non-Newtonian MHD Casson fluid flow dispersion over a radiative vertical cylinder with entropy heat generation. J. Mol. Liq. 2018, 252, 245-262. [CrossRef]

42. Afridi, M.I.; Qasim, M.; Khan, I. Entropy Generation Minimization in MHD Boundary Layer Flow over a Slendering Stretching Sheet in the Presence of Frictional and Joule Heating. J. Korean Phys. Soc. 2018, 73, 1303-1309. [CrossRef]

43. Kumam, P.; Shah, Z.; Dawar, A.; Rasheed, H.U.; Islam, S. Entropy Generation in MHD Radiative Flow of CNTs Casson Nanofluid in Rotating Channels with Heat Source/Sink. Math. Probl. Eng. 2019, 2019, 1-14. [CrossRef]

44. Saleem, S.; Nadeem, S. Theoretical analysis of slip flow on a rotating cone with viscous dissipation effects. J. Hydrodyn. 2015, 27, 616-623. [CrossRef]

45. Shah, Z.; Islam, S.; Ayaz, H.; Khan, S. Radiative Heat and Mass Transfer Analysis of Micropolar Nanofluid Flow of Casson Fluid between Two Rotating Parallel Plates with Effects of Hall Current. ASME J. Heat Transf. 2019, 141, 022401-1. [CrossRef]

46. El-Aziz, M.A.; Afify, A.A. MHD Casson Fluid Flow over a Stretching Sheet with Entropy Generation Analysis and Hall Influence. Entropy 2019, 21, 592. [CrossRef]

(C) 2020 by the authors. Licensee MDPI, Basel, Switzerland. This article is an open access article distributed under the terms and conditions of the Creative Commons Attribution (CC BY) license (http://creativecommons.org/licenses/by/4.0/). 